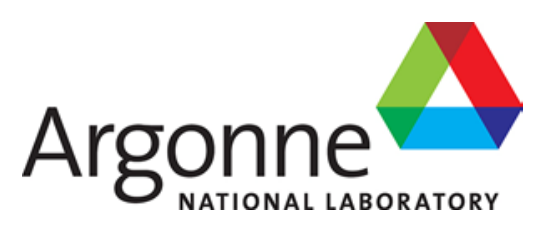

ANL-20/44

\title{
2019 LDRD Annual Report
}

Laboratory Directed Research and Development Program

Laboratory Directed Research and Development 


\begin{abstract}
About Argonne National Laboratory
Argonne is a U.S. Department of Energy laboratory managed by UChicago Argonne, LLC under contract DE-AC02-06CH11357. The Laboratory's main facility is outside Chicago, at 9700 South Cass Avenue, Argonne, Illinois 60439. For information about Argonne and its pioneering science and technology programs, see www.anl.gov.
\end{abstract}

\title{
DOCUMENT AVAILABILITY
}

Online Access: U.S. Department of Energy (DOE) reports produced after 1991 and a growing number of pre-1991 documents are available free at OSTI.GOV (http://www.osti.gov/), a service of the U.S. Dept. of Energy's Office of Scientific and Technical Information

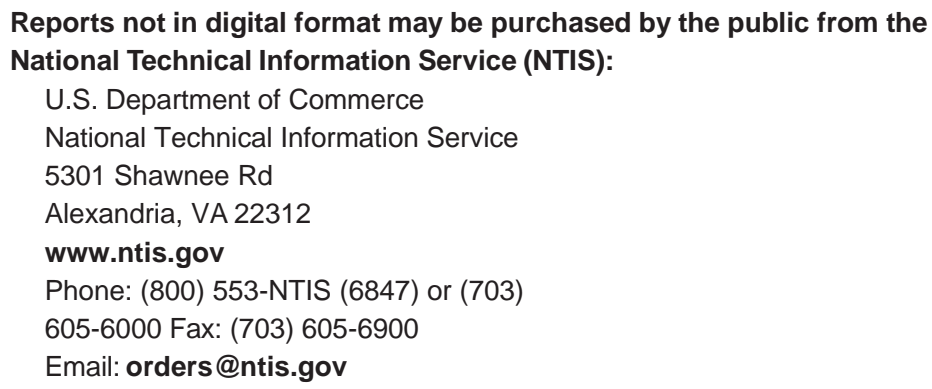

Reports not in digital format are available to DOE and DOE contractors from the Office of Scientific and Technical Information (OSTI):

U.S. Department of Energy

Office of Scientific and Technical Information

P.O. Box 62

Oak Ridge, TN 37831-0062

www.osti.gov

Phone: (865) 576-8401

Fax: (865) 576-5728

Email: reports@osti.gov

\section{Disclaimer}

This report was prepared as an account of work sponsored by an agency of the United States Government. Neither the United States Government nor any agency thereof, nor UChicago Argonne, LLC, nor any of their employees or officers, makes any warranty, express or implied, or assumes any legal liability or responsibility for the accuracy, completeness, or usefulness of any information, apparatus, product, or process disclosed, or represents that its use would not infringe privately owned rights. Reference herein to any specific commercial product, process, or service by trade name, trademark, manufacturer, or otherwise, does not necessarily constitute or imply its endorsement, recommendation, or favoring by the United States Government or any agency thereof. The views and opinions of document authors expressed herein do not necessarily state or reflect those of the United States Government or any agency thereof, Argonne National Laboratory, or UChicago Argonne, LLC. 


\section{LDRD Annual Report}

\section{Laboratory Directed Research and Development Program}

prepared by

Vivian Sullivan, LDRD Program Manager, Office of Strategy, Performance, and Risk;

Office of the Director

Laboratory Directed Research and Development, Argonne National Laboratory

August 12, 2020 


\title{
LDRD Program Overview
}

Argonne National Laboratory's Laboratory Directed Research and Development (LDRD) program encourages the development of novel technical concepts, enhances the Laboratory's research and development (R\&D) capabilities, and enables pursuit of strategic laboratory goals.

Argonne's LDRD projects are proposal based and peer reviewed, supporting ideas that require advanced exploration so they can be sufficiently developed to pursue support through normal programmatic channels. Among the aims of the projects supported by the LDRD program are the establishment of engineering proofs of principle, assessment of design feasibility for prospective facilities, development of instrumentation or computational methods or systems, and discoveries in fundamental science and exploratory development.

All LDRD projects have demonstrable ties to one or more of the science, energy, environment, and national security missions of the U.S. Department of Energy (DOE) and its National Nuclear Security Administration (NNSA), and many are also relevant to the missions of other federal agencies that sponsor work at Argonne. A natural consequence of the more "applied" type projects is their concurrent relevance to industry.

The LDRD program is managed in overarching portfolios, each containing multiple projects each fiscal year. The LDRD Prime portfolio is further divided into strategic focus areas aligned with Argonne's strategic plan.

\section{FY 2019 LDRD Program Components}

\section{LDRD Prime}

The largest component of Argonne's program is LDRD Prime, which emphasizes R\&D explicitly aligned with Laboratory major initiatives in support of Argonne's strategic plan. The choice of Focus Areas under the LDRD Prime component reflects the major initiatives; the state of development of relevant technical fields; the potential value of advancing those fields to DOE/NNSA and the nation; and the compatibility of the fields with existing facilities, capabilities, and staff expertise at Argonne.

Focus Areas with projects that ended in FY19 are:

\author{
Advanced Computing \\ Biological and Environmental Science Capability Development \\ Hard X-ray Sciences \\ Manufacturing Science and Engineering \\ Materials and Chemistry \\ National and Global Security \\ Photon Sciences \\ Securing Energy and Critical Resources \\ The Universe as Our Laboratory (ULab)
}




\section{Director's Collaborations}

The Director's Collaborations LDRD projects support research that is paired with coordinating research efforts at a partner institution. These are generally small projects selected through a collaborative process.

\section{Named Fellows}

Argonne's LDRD Named Fellows program aims to support the scientific or engineering research of exceptional early career scientists and engineers. Working with an Argonne sponsor (a senior member of research staff), LDRD Named Fellows carry out work that is either at the forefront of new research areas or is synergistic with current research efforts.

\section{Innovate}

The Innovate component of the LDRD program invests in a full spectrum of investigator-initiated proposals across the Laboratory in DOE-mission-related science and engineering areas. This provides an avenue for R\&D staff to propose highly innovative projects in research areas outside the purview of the Prime Focus Areas. Within the Innovate LDRD portfolio is the Seed program, open to postdoctoral appointees and early career researchers to apply for a small project to perform independent research and explore their own ideas.

\section{Swift}

The LDRD Swift component provides an avenue for R\&D staff to conduct short-term research with a targeted funding opportunity in mind, as well as a means for researchers to explore ideas before developing a full proposal. Projects funded through this component area have a maximum one-year duration. As with the Innovate component, the Swift component invests in a full spectrum of proposals across all mission-related science and engineering areas.

\section{FY 2019 LDRD Summary Report}

This summary report provides an overview of all LDRD projects at Argonne that concluded in Fiscal Year 2019. Many projects are funded for multiple years, the initial fiscal year for each project is indicated by the first four digits of the LDRD project number.

This material is based upon work supported by Laboratory Directed Research and Development (LDRD) funding from Argonne National Laboratory, provided by the Director, Office of Science, of the U.S. Department of Energy under Contract No. DE-AC02-06CH11357. 


\begin{tabular}{|c|c|c|c|}
\hline $\begin{array}{l}\text { LDRD Project } \\
\text { Number }\end{array}$ & Title & Lead PI(s) & Page \\
\hline \multicolumn{4}{|c|}{ ADVANCED COMPUTING } \\
\hline 2018-031 & $\begin{array}{l}\text { DLHub: Building an Open Platform to Advance and } \\
\text { Simplify Deep Learning Adoption }\end{array}$ & Ian Foster & 1 \\
\hline $\begin{array}{l}\text { 2019-0464 } \\
\text { BIOLOGICAL }\end{array}$ & $\begin{array}{l}\text { Advanced Computing Expedition } 2019 \\
\text { AND ENVIRONMENTAL SCIENCE CAPABILITY DE }\end{array}$ & Michael Papka & 1 \\
\hline 2017-087 & $\begin{array}{l}\text { Linking Climate to Water: Implementing a 4KM } \\
\text { Regional Climate Model with Hydrologic Model } \\
\text { Coupling (WRF-Hydro) using Argonne }\end{array}$ & Rao Kotamarthi & 2 \\
\hline \multicolumn{4}{|c|}{ HARD X-RAY SCIENCES } \\
\hline 2017-073 & $\begin{array}{l}\text { Developing Advanced Coherent Surface Scattering } \\
\text { Reconstruction Method Incorporating Dynamical } \\
\text { Scattering Theory }\end{array}$ & Jin Wang & 3 \\
\hline 2017-080 & COHED: Coherence for High-Energy Diffraction & Youssef Nashed & 3 \\
\hline 2017-091 & $\begin{array}{l}\text { Development of a Compact Accelerator for A High } \\
\text { Repetition Rate Free-Electron Laser }\end{array}$ & Sasha Zholents & 4 \\
\hline \multicolumn{4}{|c|}{ MANUFACTURING SCIENCE AND ENGINEERING } \\
\hline 2018-011 & $\begin{array}{l}\text { Enabling Flame Spray Pyrolysis as a Transformational } \\
\text { Manufacturing Technology }\end{array}$ & Joseph Libera & 5 \\
\hline 2018-022 & $\begin{array}{l}\text { Continuous Flow Synthesis: A Platform to Accelerate } \\
\text { the Transition of Nanomaterials to Manufacturing }\end{array}$ & Krzysztof Pupek & 5 \\
\hline 2019-0145 & $\begin{array}{l}\text { Sensor-based Mixed Reality for Rapid Digital } \\
\text { Prototyping }\end{array}$ & Young Soo Park & 6 \\
\hline \multicolumn{4}{|c|}{ MATERIALS AND CHEMISTRY } \\
\hline 2017-092 & Quantum Optics with Phonons & Andrew Cleland & 7 \\
\hline \multicolumn{4}{|c|}{ NATIONAL AND GLOBAL SECURITY } \\
\hline 2017-076 & Miniaturized High-Efficiency RF Energy Harvesting & Xing Chen, Kaizhong Gao & 7 \\
\hline \multicolumn{4}{|c|}{ PHOTON SCIENCES } \\
\hline 2017-153 & $\begin{array}{l}\text { Soft Matter Visualization and Characterization by } \\
\text { Electron Optical Beam Lines }\end{array}$ & Nestor Zaluzec & 8 \\
\hline \multicolumn{4}{|c|}{ PHYSICAL SCIENCES AND ENGINEERING } \\
\hline 2019-0296 & Fighting Cancer with Accelerators & Jerry Nolen & 9 \\
\hline \multicolumn{4}{|c|}{ SECURING ENERGY AND CRITICAL RESOURCES } \\
\hline 2018-088 & $\begin{array}{l}\text { Integrated Simulation Capabilities for Versatile Test } \\
\text { Reactor Design and Analysis }\end{array}$ & Acacia Brunett & 9 \\
\hline
\end{tabular}


2018-090

Strategic Assessment of Future Energy Needs and Emerging Technologies (SAFE-NET)

2018-093 Real-Time Characterization of VTR Coolant Variables by High Resolution Distributed Sensing

2018-095 Towards the Creation of an Advanced Mobility Cybersecurity Testbed

\section{THE UNIVERSE AS OUR LABORATORY (ULAB)}

2017-058 A Strategic Scientific Program to Establish Argonne Leadership in the Development of the Future Electron-Ion Collider

2018-012 A High Precision Superconducting Detector for Next Generation Neutrinoless Double Beta Decay Search Experiments

\section{DIRECTOR'S COLLABORATIONS}

\section{7-156 Scalable Data Movement for Data-centric} Supercomputing

2018-106

Synergistic Multi-Site Catalysts For Dry Reforming Processes

2018-107 Creating Next-Generation Quantum Electronics with Topological Insulator/Ferromagnetic Insulator Heterostructures

2018-116 Long-Lived Rare-Earth Qubits on Silicon

2018-117 Dynamic Cross-linked Polymers for Silicon-based Electrodes in Lithium-Ion Batteries

2019-0383 Sustainability of Brackish Groundwater in the MidContinental Region

\section{NAMED FELLOWS}

2016-183 Understanding and Controlling Charge, Spin, Pseudospins and Lattice Degrees of Freedom in Layered Transition Metal Dichalcogenides

2017-031 Ecological Organic Photovoltaics using Water-borne Semiconductor Nanoparticles

2017-094 Microstructural Simulations of Stable Conjugated Polymer Glasses

2017-097

2018-111

2018-112 A Universal Data Analytics Platform for Science Bio-inspired Smart Soft-Robots Engineered Materials for Additive Manufacturing

\section{INNOVATE}

2017-019

2017-026
Bonding Dissimilar Materials using Nanoparticles/Nanofilm as Eutectic Compounds Realizing a Gate Tunable Kinetic Inductance for a Transmon Qubit using SrTiO3
Charles Macal

Darius Lisowski

Roland Varriale

Kawtar Hafidi, Jose

12 Repond

Gensheng Wang

Venkatram Vishwanath

14

Massimiliano Delferro

14

John Freeland

15

Supratik Guha

16

Daniel Abraham

Peter Mueller

Qi Zhang

Yu Jin Kim

Nicholas Jackson

Ryan Chard

20

Xu Zhang

20

Sarah Wolff

Kamleshkumar Suthar

Anand Bhattacharya 
2019-0006

2019-0010

2019-0024

2019-0048

2019-0057

2019-0083

2019-0100

2019-0104

2019-0112

2019-0142

2019-0153

2019-0246

2019-0292

2019-0316

SWIFT

2019-0392

2019-0395

2019-0397

2019-0398

2019-0404

2019-0408
Deep Learning for Posterior Density Emulation and Sampling

Large-Scale Uncertainty Quantification for Power System Resilience

Krypton-85 Analysis in Uranium Hexafluoride via Atom Trap Trace Analysis

A New View on Charge and Spin in Functional Metal Oxides

Development of an Integrated, Mechanistic

Framework for Lead-Cooled Fast Reactor Source Term Assessments

Production of Chemically and Mineralogically

Accurate Far-Field Fall Out Particles Using an Electric

Arc Furnace

FTK: The Feature Tracking Kit

Computational Framework to Characterize Extreme Events for Complex Physical Phenomena

Goal-Oriented Optimal Experimental Design

Framework for Sensor Placement and Acquisition of Highly-Correlated Data

Statistical Multi-Scale Methods for Parameterization of Complex Physics-Based Models

Fabrication of Single Crystal and High Aspect Ratio

Piezoelectric Nanofibers for Nanogenerator

Applications

Developing Capability to Generate High Resolution

Agronomic and Economic Decision Variables for

Creating Win-Win for Bioenergy Crops and Water

Quality

Computational Design of Li-ion Cathodes Based on

Transition Metal Oxysulfide

Magnetically-Enforced Microbial Scavengers

Enhancing Simulation Tools for Hypersonic Flight

Through Improved Fuel Injection Modeling in Air-

Breathing Jet Engines

Generation of a Next Level Dataset for Regional Scale

Climate Modeling: Convective Resolving Spatial Scales

Combining High-Performance Computing, Artificial

Intelligence, and Agent-Based Models to Combat

Misinformation Campaigns

Laser Joining of Dissimilar Metals

Design of a Modular Ocean Mining System for

Seawater Rare Element Extraction and Desalinization

Advanced Moderation Module (AMM) Development

for Micro-Very-High-Temperature Reactors
Carlo Graziani

Michel Schanen

Peter Mueller

24

Alexander Rettie

24

Matthew Bucknor

Derek McLain

26

Hanqi Guo 26

Vishwas Rao 27

Ahmed Attia

27

Julie Bessac

Moinuddin Ahmed

28

Shruti Khadka Mishra

Liang Li

29

Gasper Kokot

Gina Maureen Magnotti

Jiali Wang

Matthew Wolf

Xuan Zhang

Mark Christian

Yinbin Miao 


\begin{tabular}{|c|c|c|}
\hline 2019-0409 & Open Source Atmospheric Data Community Toolkit & Adam Theisen \\
\hline 2019-0410 & $\begin{array}{l}\text { A Novel Security Analysis Toolbox for National Grid } \\
\text { Resilience Modeling }\end{array}$ & Feng Qiu \\
\hline 2019-0412 & State Preservation for Deep Learning Applications & Bogdan Nicolae \\
\hline 2019-0437 & $\begin{array}{l}\text { An Internet Islanding Approach to Deterrence of } \\
\text { Nation-State Adversaries }\end{array}$ & Nate Evans \\
\hline 2019-0481 & $\begin{array}{l}\text { Cathode Composite Electrode Composed of } 1 \text { Micron- } \\
\text { size Single Crystal LLZO Filler and Core(NMC811)- } \\
\text { Shelled(LLZO) Cathode }\end{array}$ & YoungHo Shin \\
\hline 2019-0482 & $\begin{array}{l}\text { Development of Dense LLZO Solid-State Electrolytes } \\
\text { from Ultrafine-Grain Nanofibers }\end{array}$ & Yuepeng Zhang \\
\hline 2019-0483 & $\begin{array}{l}\text { Aerosol Deposition of Dense Ceramic Layers for All- } \\
\text { Solid-State Batteries }\end{array}$ & Eungje Lee \\
\hline 2019-0486 & $\begin{array}{l}\text { Rapid Microwave-Assisted Sintering of LLZO Solid } \\
\text { State Electrolyte: An Energy-Efficient Manufacturing } \\
\text { Alternative }\end{array}$ & Jessica Durham \\
\hline 2019-0487 & $\begin{array}{l}\text { Development of Conductive Solid-State LLZO } \\
\text { Electrolyte by Hot-pressing of Electrospun Precursor } \\
\text { LLZO Nanofibers }\end{array}$ & Dileep Singh \\
\hline 2019-0494 & $\begin{array}{l}\text { Manufacturing Processes for Lithium Metal Using } \\
\text { Advanced Electrodeposition }\end{array}$ & Edward Barry \\
\hline 2019-0498 & $\begin{array}{l}\text { Fabrication of Large Area Dense Separator via Tape } \\
\text { Casting and Pressure-less Sintering }\end{array}$ & Xiaoping Wang \\
\hline $2019-0501$ & $\begin{array}{l}\text { Generating Training Datasets for Developing a Deep } \\
\text { Learning-Computer Vision Framework to Monitor } \\
\text { Avian Interactions with Solar Energy Facility } \\
\text { Infrastructure }\end{array}$ & Leroy Walston \\
\hline 2019-0506 & CFD modeling of Rotating Detonation Engines (RDE) & Sibendu Som \\
\hline 2019-0507 & Data Enabled Catalyst Discovery & Rajeev Surendran Assary \\
\hline 2019-0508 & $\begin{array}{l}\text { Al-Coupled Experiment and Simulation to Create } \\
\text { Tailored 3D Auxetic Materials }\end{array}$ & Benjamin Blaisik \\
\hline 2019-0509 & $\begin{array}{l}\text { Al Robotic Platform for Solution-Processable } \\
\text { Materials }\end{array}$ & Jie Xu \\
\hline $2019-0510$ & $\begin{array}{l}\text { Study of Potential Applications to Ultrafast Electron } \\
\text { Diffraction/Microscopy Using a High Repetition Rate } \\
\text { Superconducting Electron Photocathode Gun }\end{array}$ & John Byrd \\
\hline
\end{tabular}




\section{ADVANCED COMPUTING}

\begin{tabular}{|l|l|}
\hline LDRD\# 2018-031 & Advanced Computing \\
\hline Project Title: & $\begin{array}{l}\text { DLHub: Building an Open Platform to Advance and Simplify Deep Learning } \\
\text { Adoption }\end{array}$ \\
\hline Investigators: & $\begin{array}{l}\text { lan Foster } \\
\text { Benjamin Blaisik }\end{array}$ \\
\hline
\end{tabular}

\section{Abstract}

Scientists are increasingly limited by their ability to integrate, model, and act on data. This is particularly apparent in materials science and chemistry, where scientists generate large amounts of experimental data from many user facilities and computational data from simulations at leadership computing facilities. Additional data are also accessible from external repositories to supplement those generated locally. Development of new machine learning and, especially, of deep learning (ML/DL) capabilities creates an opportunity to integrate data streams and to allow ML/DL techniques to guide and even lead discovery efforts. Challenges include data in disparate locations and in heterogeneous formats, disparate application programming interfaces (APIs), and authentication barriers. Also, many researchers today lack the necessary expertise fully utilize required computational tools, models, and algorithms. In any case, the lack of open platforms for storing, deploying, sharing, and using models can lead to duplicated effort. In this project, we tackle these roadblocks by building a self-service platform, DLHub, for publishing, applying, and creating new ML/DL models that will help new data science approaches and models translate more immediately into actual improvements in practice. We initially targeted specific cases in materials science and chemistry. DLHub provides (1) publication capabilities to make models more discoverable, citable, and reusable; (2) the ability to easily run or test existing models; and (3) links to the data and computing infrastructure to re-train models for new applications. Extensive components of the DLHub have been implemented including: Architecture as a cloud-hosted service; Model schema to standardize model definitions and ensure all necessary data for deployment and publication are captured; Model ingest workflow to accommodate self-publication of models; Simplified pipelines for predicting material properties including prediction of metallic glass forming compositions; and Batch classification of X-ray scattering data.

\begin{tabular}{|l|l|}
\hline LDRD\# 2019-0464 & Advanced Computing \\
\hline Project Title: & Advanced Computing Expedition 2019 \\
\hline Investigators: & $\begin{array}{l}\text { Michael Papka } \\
\text { lan Foster, Salman Habib, Valerie Taylor }\end{array}$ \\
\hline
\end{tabular}

\section{Abstract}

Our project is a fast-track, focused effort to explore the use of new computing technologies to solve problems of interest to Argonne and DOE. This effort provides a rapid deep-dive into computing technologies related to Argonne domain science. Our investigators explored a quantum algorithm or a neuromorphic application, the use of accelerator technology for deep learning, and collaboration to optimize a code for the Aurora exascale system. Nineteen "expeditions" are supported across these 
areas in this umbrella project. These expeditions allow for exploratory looks at a number of different areas and at how they can be enabled by future computing technologies. Specifically, the following 19 topics were studied: Quantum Molecular Dynamics; Efficient Classical Simulation of Quantum Circuits; Investigating Quantum Chemistry Circuits with OpenFermion; Performance Study of Quantum Algorithm Implementations in Quac; Develop Quantum Algorithms to Simulate Higher Order LHC Interactions; Modeling Quarks on Quantum Computers; Evaluating Deep Neural Network to Spiking Neural Networks Transformation for DOE Scientific Applications on Intel Loihi; Neuromorphic Classification of High Energy Physics Data; Enabling an Image Recognition DSK Module Using Neural Network on Neuromorphic Chips; Prototyping a Sensory Model of Cyber Situational Awareness for Anomaly Detection; Earthquake Forecasting with Liquid State Machines; Neuromorphic Computing for Feature Extracting and Tracking; Exploring Performance and Power of Parallel CANDLE Benchmarks on Accelerator Technology for Deep Learning; Accelerating Ab Initio Many-Body Computations with Al-Purposed Hardware via MixedPrecision Iterative Refinement; Accelerating Hierarchical Recurrent Attention Neural Networks for Deep Proteomics and Text Comprehension; Tomographic Reconstruction with Accelerator Technology and Deep Learning; Benchmark and Optimization of 3D-FFT Solvers for Many-Body Perturbation Theory Calculations; A21 Accelerators for Advancing Optimization Solvers; and High-Order Derivative Computation on Advanced Computer Architectures.

\section{BIOLOGICAL AND ENVIRONMENTAL SCIENCE CAPABILITY DEVELOPMENT}

\begin{tabular}{|l|l|}
\hline LDRD\# 2017-087 & Biological and Environmental Science Capability Development \\
\hline Project Title: & $\begin{array}{l}\text { Linking Climate to Water: Implementing a 4KM Regional Climate Model with } \\
\text { Hydrologic Model Coupling (WRF-Hydro) using Argonne }\end{array}$ \\
\hline Investigators: & $\begin{array}{l}\text { Rao Kotamarthi } \\
\text { Andrew Orr, Beth A. Drewniak, Cheng Wang, Jiali Wang, John Krummel, } \\
\text { Preeti Malakar, Randall Gentry, Venkatram Vishwanath }\end{array}$ \\
\hline
\end{tabular}

\section{Abstract}

A fundamental aspect of addressing the energy-water-land (EWL) nexus is the need to develop multiscale analysis and assessment tools for the three sectors (energy, water, and land use) and to couple them over a range of time and spatial scales. Our work is intended to provide a fully coupled climatehydrology model with the means to evaluate feedbacks among the atmosphere, land, and hydrology under current and future climate conditions. Our method will improve future land surface and climate modeling studies. Development of the fully coupled atmosphere-land-hydrology model at $4 \mathrm{~km}$ over the continental United States (CONUS) brings a layer of heterogeneity to the land surface model representing small scale variability in soil type, vegetation structure, and topography. At high resolutions, this variability becomes important, as hydrologic responses depend greatly on a model's spatial resolution. This project led to the core capacity for performing continental scale, very high spatial resolution (200 meters) simulations of surface flow using the community model WRF-Hydro. We developed a parallel calibration capability to tune the model over a large number of grid locations on high-performance computing (HPC) clusters and enabled use of the model over large spatial domains. We have also established the feasibility of running this model over decadal length scales at large regional and continental spatial scales. By scaling the model to HPC, we are now able to perform risk 
assessments of change in flood level with changing climate in mid-century conditions compared to the present.

\section{HARD X-RAY SCIENCES}

\begin{tabular}{|l|l|}
\hline LDRD\# 2017-073 & Hard X-ray Sciences \\
\hline Project Title: & $\begin{array}{l}\text { Developing Advanced Coherent Surface Scattering Reconstruction Method } \\
\text { Incorporating Dynamical Scattering Theory }\end{array}$ \\
\hline Investigators: & $\begin{array}{l}\text { Jin Wang } \\
\text { Tao Sun, Wei Jiang, Zhang Jiang }\end{array}$ \\
\hline
\end{tabular}

\section{Abstract}

Coherent surface scattering imaging (CSSI) has unique advantages for exploring surface and interface structures and their dynamics when they are responding to external stimuli. The implementation of the CSSI technique requires state-of-the-art image reconstruction. This project's goal was to develop a novel scattering-physics-based reconstruction method that incorporates dynamical scattering theory in the grazing-incidence condition, enabling CSSI to be a true 3D structural probe for surface or interface visualization. The reconstruction of coherent diffractive images has to date required phase retrieval to complement X-ray scattering intensity measurements using a partially coherent beam. However, those algorithms use kinematic theory, which assumes that the scattering from the sample is weak. With that assumption, the reconstruction is only a mathematical process involving recursive fast Fourier transform (FFT) and its inverse. These algorithms cannot be simply applied to the CSSI case where the weak scattering assumption is not satisfied at the grazing angles. Hence, we developed a new surface coherent scattering algorithm that calculates and simulates $x$-ray surface scattering data, taking into account the dynamical effects of scattering at low scattering angles. We optimized and deployed the algorithm incorporating a grid-DWBA (distorted-wave Born approximation) at the Argonne Leadership Computing Facility (ALCF) so that the reconstruction will become quasi real time to help experimental decisions at the beamline. Our algorithm will be deployed for data analysis and reconstruction at one of the beamlines at the APS-U when commissioned in 2023.

\begin{tabular}{|l|l|}
\hline LDRD\# 2017-080 & Hard X-ray Sciences \\
\hline Project Title: & COHED: Coherence for High-Energy Diffraction \\
\hline Investigators: & $\begin{array}{l}\text { Youssef Nashed } \\
\text { Jonathan Almer, Jun-Sang Park, Peter Kenesei, Ross Harder, } \\
\text { Siddharth Maddali Vivekanand, Stephan Hruszkewycz }\end{array}$ \\
\hline
\end{tabular}

\section{Abstract}

High-energy diffraction microscopy (HEDM) and coherent diffraction imaging (CDI) have a proven ability, respectively, to collect detailed strain images at nanometer resolutions on single grains and at micrometer resolutions on thousands of grains. This project combines these techniques, which we term Coherence for High-Energy Diffraction (COHED). We primarily focused on the problem of three- 
dimensional image reconstruction from high-energy coherent x-ray Bragg diffraction patterns. First, we performed preliminary simulations of the effects of high-energy $X$-rays in a Bragg coherent diffraction imaging ( $B C D I$ ) experiment, which helped to find the limit of current state-of-the-art phase retrieval algorithms under conditions relevant to high-energy BCDI. On the algorithmic side, we developed a sparsity-based approach in which fine fringe features can be recovered from over-pixelated patterns, a formalism to handle partial coherence specifically for the case of high energy BCDI, and a BCDI modulus constraint for direct phasing of under-sampled 3D BCDI data sets. For experimental validation, we reconstructed the first ever 3D BCDI image of both an isolated nanocrystal and a single grain within a polycrystalline film. We obtained 3D reconstructions of a single Au nanocrystal from data measured with a fully coherent beam under conditions of over- and under-sampling. Anticipating the APS Upgrade, COHED will be integrated into the HEDM imaging capabilities at the APS and will rely on computational resources for iterative reconstruction of terabyte-scale data sets.

\begin{tabular}{|l|l|}
\hline LDRD\# 2017-091 & Hard X-ray Sciences \\
\hline Project Title: & $\begin{array}{l}\text { Development of a Compact Accelerator for A High Repetition Rate Free- } \\
\text { Electron Laser }\end{array}$ \\
\hline Investigators: & $\begin{array}{l}\text { Sasha Zholents } \\
\text { Emil Trakhtenberg, Geoff Waldschmidt, John Power, Kamleshkumar Suthar, } \\
\text { Matthew Kasa, Scott Doran, William Jansma, Yine Sun }\end{array}$ \\
\hline
\end{tabular}

\section{Abstract}

The goal of this project is an in-depth assessment of the feasibility of a novel accelerator technology based on the design of a compact collinear wakefield accelerator (CWA) and on the prototyping and testing of its components. We focus on the development and validation of a few key elements of a compact accelerator that present high technical risks. Our work anticipates the opportunity presented by a new accelerator technology under development for a linear collider that will enable the construction of high-brightness, high-repetition-rate accelerators at a potentially significantly reduced scale and cost. If successful, the new technology will make x-ray free electron lasers (XFELs) considerably more affordable and create an opportunity to build one at Argonne National Laboratory. We envision multiple FEL-based beamlines, where each FEL will have its own designated high-repetition-rate compact wakefield accelerator. The electron bunches will be fed to each compact accelerator from a common high-repetition-rate superconducting linac. The main CWA component is a copper tube with a $2 \mathrm{~mm}$ internal diameter and small corrugations on the internal wall. We have made an extensive study of various ways to manufacture and test this component, both by prototyping and by simulating its performance. The accelerator design is optimized to yield about a $100 \mathrm{MeV} / \mathrm{meter}$ rate of the energy gain and to accelerate electrons in the trailing bunch from $1 \mathrm{GeV}$ to $5 \mathrm{GeV}$. The accelerator can support up to a $50 \mathrm{kHz}$ bunch repetition rate. We have also designed a quadrupole wiggler to surround the accelerator tube for beam stability. Our design of an ultra-compact transition region between accelerator modules includes an output rf coupler, a notch filter, an integrated offset monitor, bellows, and pumping and water-cooling ports. The integrated offset monitor is a novel diagnostic that measures the cumulative offset of the electron beam in the tube with corrugations upstream of the monitor. The design of most of the accelerator components has reached a pre-construction phase. We began 
fabrication of a half-meter-long accelerator module for a test with the electron beam at the Argonne Wakefield Accelerator facility.

\section{MANUFACTURING SCIENCE AND ENGINEERING}

\begin{tabular}{|l|l|}
\hline LDRD\# 2018-011 & Manufacturing Science and Engineering \\
\hline Project Title: & $\begin{array}{l}\text { Enabling Flame Spray Pyrolysis as a Transformational Manufacturing } \\
\text { Technology }\end{array}$ \\
\hline Investigators: & $\begin{array}{l}\text { Joseph Libera } \\
\text { Jan Ilavsky, Karena Chapman, Robert Tranter, Theodore Krause }\end{array}$ \\
\hline
\end{tabular}

\section{Abstract}

Flame spray pyrolysis (FSP) is an emerging manufacturing technology for the rapid and scalable production of materials ranging from simple metal oxides to complex multicomponent metal oxides and oxide-supported metals-materials found in heterogeneous catalysts. FSP occurs at high temperature with intimate mixing of precursor compounds which undergo combustion, leading to particle growth. Rapid quenching can produce metastable phases, yielding materials with physiochemical properties that are typically employed in catalyst manufacturing. The technology could be more widely adopted for producing complete catalysts. The fundamental reaction mechanisms, reaction kinetics, and transport processes that govern particle growth need to be understood. An FSP facility at Argonne's Materials Engineering Research Facility (MERF) gives us an opportunity to pursue a fundamental understanding of FSP, one that will allow wider adoption. In-situ diagnostics combined with synthesis of new families of catalysts that were discovered on the bench scale will open new pathways to future methods for synthesis of important catalytic materials. We have successfully addressed many aspects of this study including burner commissioning and optimization, scanning mobility particle sizing (SMPS), FSP solids production, studies of $\mathrm{Fe}-\mathrm{SiO}_{2}$ and $\mathrm{Zn}-\mathrm{Pt} / \mathrm{SiO}_{2}$ catalysts, in-situ sampling for APS (for USAXS/SAXS), laser diagnostic system specification and procurement, shock tube investigations, and optical emission spectroscopy. We have provided a state-of-the-art diagnostic capability to help optimize material synthesis by FSP. The complete diagnostic ensemble has also provided a platform for the development of machine learning algorithms to solve the challenging multi-physics problem that is FSP.

\begin{tabular}{|l|l|}
\hline LDRD\# 2018-022 & Manufacturing Science and Engineering \\
\hline Project Title: & $\begin{array}{l}\text { Continuous Flow Synthesis: A Platform to Accelerate the Transition of } \\
\text { Nanomaterials to Manufacturing }\end{array}$ \\
\hline Investigators: & $\begin{array}{l}\text { Krzysztof Pupek } \\
\text { Karena Chapman, Stefan Wild, Vojislav Stamenkovic }\end{array}$ \\
\hline
\end{tabular}

\section{Abstract}

Scaling up batch-type lab syntheses to manufacture nanomaterials at the scale needed for applications such as catalysis and electrochemical energy storage is challenging because at large scales, tight control of synthesis conditions is problematic, leading to unacceptable variability in target nanomaterial 
properties. On the other hand, continuous-flow microfluidic synthesis for scaled-up manufacturing features predictable heat and mass transfer rates, safe operation under high pressures and temperatures, and precise control of conditions to deliver greater precision and manufacturing efficiency. To apply microfluidic reactors to nanomaterials manufacture, the combination of process parameters must be determined that will deliver the desired size, shape, morphology, composition and phase of the product. In this project, we demonstrated the synthesis of bimetallic platinum-nickel (PtNi) alloy nanoparticles (a fuel-cell catalyst) in a continuous flow reactor, and we established both ex-situ segmentation computer code for transmission electron microscope (TEM) analysis and in-situ and ex situ X-ray characterization methods (small angle x-ray scattering (SAXS) and pair distribution function (PDF) measurement) at the Advanced Photon Source (APS) to correlate reaction conditions with nanomaterial structural properties. The microfluidic reactor for nanoparticle synthesis was designed and engineered to allow for simultaneous SAXS and PDF measurements. While SAXS gave us insights into particle size and distribution, PDF allowed us to determine the evolution of the structure and morphology of the nanoparticles during synthesis under non-equilibrium conditions. The structural information served as input for machine-learning algorithms and data-science approaches that rely on Argonne's expertise in data science and computing. The outcome is a broadly applicable approach to rapidly and efficiently identifying optimal microfluidic process parameters for a target nanomaterial structure.

\begin{tabular}{|l|l|}
\hline LDRD\# 2019-0145 & Manufacturing Science and Engineering \\
\hline Project Title: & Sensor-based Mixed Reality for Rapid Digital Prototyping \\
\hline Investigators: & $\begin{array}{l}\text { Young Soo Park } \\
\text { Marius Stan }\end{array}$ \\
\hline
\end{tabular}

\section{Abstract}

The goal of our project is development of a mixed-reality system for rapid prototyping of a modular materials robotic platform (MMRP) and demonstration of its usefulness for automation of materials synthesis processes and more rapid materials discovery. We have an opportunity to scale-up and speed up the materials discovery process by incorporating a modular and flexible robotic system for automation and autonomous materials processing along with artificial intelligence (Al). The prototype of the MMRP provides hardware-in-the-loop simulation capability for select advanced materials processes. The Al-based approaches evaluate the feasibility of the autonomous discovery process. We first performed a thorough review of materials processes at Argonne and established our design concept. We then developed a virtual digital prototype of the MMRP. A physical prototype of the MMRP was constructed. Mixed-reality was achieved by implementing the hardware-in-the-loop operation. And, we developed an Al-based framework for human-interactive programming of the robot operation for a materials synthesis process in such a way as to extract human skills. Initial trials were applied to high throughput catalyst electrolyte cyclic voltammetry testing for battery and fuel cell development and to solution separation processes. 


\begin{tabular}{|l|l|}
\hline LDRD\# 2017-092 & Materials and Chemistry \\
\hline Project Title: & Quantum Optics with Phonons \\
\hline Investigators: & Andrew Cleland \\
\hline
\end{tabular}

\section{Abstract}

Microwave-frequency phonons have wavelengths of order 0.01 to 1.0 micrometer, comparable to the range from soft $x$-rays through visible light, but at frequencies five orders of magnitude smaller. Phonons share many of the properties of photons, exhibiting diffraction, interference, and scattering through interactions with irregularities in solids. Phonons can pass through otherwise opaque materials and can image those materials and their interfaces. This project is focused on demonstrating fundamental scientific aspects associated with classical and quantum behavior of propagating phonons in the few $\mathrm{GHz}$ frequency range. We are developing a class of "optics" structures for phonons similar to those used to guide and trap photons: waveguides, resonators, and beam splitters, combined into interferometers, having overall dimensions of a few to a few hundred micrometers. We have demonstrated some of the fundamental quantum optics processes associated with microwave frequency phonons generated and detected at milli-Kelvin temperatures, where single quantum control and detection is possible. Superconducting qubits can control and detect individual phonons in a resonant structure, enabling the coherent generation and measurement of complex stationary phonon states. We have completed quantum measurements with two such qubits coupled to a delay line with a directional interdigitated transducer (IDT). We now can successfully transfer quantum states between two qubits, enabling their quantum entanglement. A redesigned IDT has improved substantially on our initially observed transfer fidelity. This phonon frequency band also enables exploration of interactions with atomic-like structures, such as defect spins, as well as fundamental quantum optics effects.

\section{NATIONAL AND GLOBAL SECURITY}

\begin{tabular}{|l|l|}
\hline LDRD\# 2017-076 & National and Global Security \\
\hline Project Title: & Miniaturized High-Efficiency RF Energy Harvesting \\
\hline Investigators: & $\begin{array}{l}\text { Xing Chen, Kaizhong Gao } \\
\text { Ana Aragon Sanchez, Yuepeng Zhang, Yunsong Xie }\end{array}$ \\
\hline
\end{tabular}

\section{Abstract}

Ambient RF energy is available in most areas to power mobile devices and sensors, allowing them to operate in strategic, remote, or hostile environments without a battery. However, RF energy harvesting devices are currently large and inefficient. State-of-the-art RF/microwave subsystems, such as antennas, are bulky or extremely narrow-band because employed materials lack usable magnetic permeability. Recently, we have developed tunable magnetodielectric composite materials that have high ferromagnetic resonance (FMR) at microwave frequencies. We can form these materials into highaspect-ratio shapes of nanosize dimensions. Such high-permeability magnetic nanofibers enable fabrication of compact, high-efficiency, broadband micro-strip antennas. They can significantly reduce total device footprint and increase bandwidth, allowing higher energy harvest rates while reducing antenna size. Nanofibers ( $<50 \mathrm{~nm}$ diameter) insulated by an atomic layer deposition (ALD) coating 
allowed us to use high moment ferromagnetic materials such as $\mathrm{Fe}_{65} \mathrm{Co}_{35}$. The high aspect ratio magnetic nanofibers were fabricated using an integrated electrospinning and heat-treatment process. As for subsystems, the largest components are the inductors and capacitors. Here, a well-aligned CoFe magnetic nanofiber-polymer assembly formed a high-permeability substrate that reduced inductor size so it could be integrated onto a chip. Capacitor sizes were reduced using a 3D solid-state integrated capacitor formed by atomic layer deposition (ALD). The titanium nitride/hafnium oxide ( $\mathrm{TiN} / \mathrm{HfO}_{2} / \mathrm{TiN}$ ) metal-dielectric insulator-metal multilayer was deposited to form the capacitor stack.

\section{PHOTON SCIENCES}

\begin{tabular}{|l|l|}
\hline LDRD\# 2017-153 & Photon Sciences \\
\hline Project Title: & Soft Matter Visualization and Characterization by Electron Optical Beam Lines \\
\hline Investigators: & Nestor Zaluzec \\
\hline
\end{tabular}

\section{Abstract}

Electron optical systems are a well-established imaging technology for characterizing structures at the micro-, nano-, and atomic scales. It is important to image not only hard (inorganic) materials, but also soft/hard interfaces and purely soft matter (SM) systems, including in their native environments rather than in vacuum. Our project expands Argonne's role in the study of SM systems. We assessed the limits of Argonne's resources as they pertain to traditional scanning/transmission electron microscopy; explored the feasibility of new opportunities presented by advanced technologies, such as pixelated array detectors, in-situ environments, and structured probes; and developed a suite of experimental methodologies tailored to SMs for measurements under minimally invasive conditions. The major challenge in soft matter systems is the ability to establish artifact free imaging/spectroscopy conditions with sufficient signal/contrast. To accomplish SM characterization, it was necessary to conduct quantitative measurements of electron scattering and associated secondary signals. We identified real world specimens (organic membranes, photo-proteins, bacteria, and amorphous silicates) to test the limits of the methods. Correlative experiments between the bionanoprobe (BNP) and electron microscopy (EM) beam lines were conducted to quantitatively measure relative performance. We completed the design and had fabricated a beryllium cryo-transfer, cryo-tomographic, cryospectroscopy soft matter holder. Lastly, a new protocol for assessing the relative merits of electron vs $\mathrm{X}$ ray spectroscopy has been established. 


\begin{tabular}{|l|l|}
\hline LDRD\# 2019-0296 & Physical Sciences and Engineering \\
\hline Project Title: & Fighting Cancer with Accelerators \\
\hline Investigators: & Jerry Nolen \\
& $\begin{array}{l}\text { Shaofei Zhu, Amanda Youker, Jeongseog Song, Matthew Gott, } \\
\text { Brahim Mustapha, Dave Rotsch, John P. Greene, Michael Alex Brown }\end{array}$ \\
\hline
\end{tabular}

\section{Abstract}

One of the highest priority radioisotopes specified for development by the Office of Nuclear Physics Isotope Program for use in cancer therapy is the short-lived alpha particle emitter Astatine-211 $\left({ }^{211} \mathrm{At}\right)$. It can be used for radio-labelling of agents such as mono-clonal antibodies. Preliminary human trials have been promising, but there is limited availability of this therapeutic isotope. It has a half-life of only seven hours, making it difficult to distribute beyond where it is produced. We have proposed that an effective way to extend the effective lifetime is to bombard a bismuth (Bi)-bearing target with a lithium ion beam to make the longer-lived isotope, Radon-211 $\left({ }^{211} \mathrm{Rn}\right)$, which decays via positron emission to ${ }^{211} \mathrm{At}$. Our goal is to use this reaction, ${ }^{209} \mathrm{Bi}\left({ }^{7} \mathrm{Li},{ }^{5} \mathrm{n}\right)^{211} \mathrm{Rn}$, and also to demonstrate a method of continuous extraction of ${ }^{211} \mathrm{Rn}$ gas from a target as the basis of a ${ }^{211} \mathrm{Rn} /{ }^{211} \mathrm{At}$ generator for clinical applications. Through use of such a generator, given the 14 -hour half-life of the ${ }^{211} \mathrm{Rn},{ }^{211} \mathrm{At}$ can be available anywhere in the country via overnight shipping. The Argonne Tandem Linac Accelerator System (ATLAS) is one of only two or three accelerators in the United States having the required lithium beam. We irradiated nanoporous $\mathrm{Bi}_{2} \mathrm{O}_{3}$ targets with various $\mathrm{Li}$ beam current intensities and for various exposure times. Attempts were made to extract, capture and collect the ${ }^{211} \mathrm{Rn}$ gas through heating the target and entrapment in a flowing He gas and such media as activated charcoal. The reaction yield could be quantified by monitoring the ${ }^{207} \mathrm{Bi}$ isotope (32-year half-life) which is a $50 \%$ byproduct of the radon isotope's decay (both via the At isotope alpha decay and through and intermediate polonium isotope decay product).

\section{SECURING ENERGY AND CRITICAL RESOURCES}

\begin{tabular}{|l|l|}
\hline LDRD\# 2018-088 & Securing Energy and Critical Resources \\
\hline Project Title: & Integrated Simulation Capabilities for Versatile Test Reactor Design and Analysis \\
\hline Investigators: & $\begin{array}{l}\text { Acacia Brunett } \\
\text { Rui Hu, Thomas Fanning }\end{array}$ \\
\hline
\end{tabular}

\section{Abstract}

DOE has established the Versatile Test Reactor (VTR) program to develop a flexible and economical fast neutron testing facility that applies modern, advanced diagnostics. This project is developing flexible and integrated system simulation capacity that is necessary for the VTR program. The work will close gaps in VTR design, analysis, modeling, and simulation (M\&S) capabilities. Enabling comprehensive testing under a wide range of advanced reactor conditions is the goal. Currently, integrated tools capable of mechanistic modeling of VTR and a test vehicle do not exist. To address this design and analysis deficiency, Argonne's SAS4A/SYSSYS-1 safety analysis code is being coupled with SAM (System Analysis Module) to provide a novel modeling capability that supports VTR and other fast test facilities. A range of testing has been completed to support demonstration of this interface. The testing scope included 
steady-state verification using a series of increasingly complex analytical solutions and transient demonstration for a range of design basis accident scenarios using prototypic VTR and test vehicle configurations. Results of this testing confirm verification of the steady-state solutions and provide reasonable and expected transient behavior. The coupling interface has been developed to be robust yet flexible and to support coupling to any external software or module.

\begin{tabular}{|l|l|}
\hline LDRD\# 2018-090 & Securing Energy and Critical Resources \\
\hline Project Title: & $\begin{array}{l}\text { Strategic Assessment of Future Energy Needs and Emerging Technologies } \\
\text { (SAFE-NET) }\end{array}$ \\
\hline Investigators: & $\begin{array}{l}\text { Charles Macal } \\
\text { Guenter Conzelman, Hyekyung (Clarisse) Kim }\end{array}$ \\
\hline
\end{tabular}

\section{Abstract}

Because the future energy landscape will be fundamentally different from that of today, new forecasting tools and methods for understanding the evolution of the energy system are needed based on the latest developments in modeling and simulation combined with emerging data analytics and machine learning techniques. An integrated framework that envisions and evaluates potentially disruptive forces would enable us to understand future scenarios for the energy system, inform strategic investments in energy technology R\&D, and identify disruptive technologies and new pathways toward energy security and sustainability. This Strategic Assessment of Future Energy Needs and Emerging Technologies (SAFE-NET) project creates a comprehensive platform that embodies all aspects of the energy system, related resources, supply chains, fuel cycles, and energy technologies. By combining technology characterizations with infrastructure models, geospatially articulated supply chain representations, and business and consumer decision-making based on agent-based modeling approaches, SAFE-NET addresses key questions about future energy systems under multiple scenarios. To develop SAFE-NET, energy systems were analyzed across all components of the energy system, energy data were compiled for all aspects of the energy system and for all current and future energy technologies that were identified in the literature, the state-of-the-art in the development of energy storage technologies was identified and characterized, and a comprehensive study was made of all energy futures studies developed by government and industry sources. One outcome of our work is the ability to extract technology options, performance requirements, and deployment scenarios. This informs regulatory decisions that include addressing flexibility and resilience of the overall energy system. The value proposition of emerging technologies, such as energy storage and advanced modular reactors, and related research needs for materials and associated manufacturing processes are also informed by outputs of the SAFE-NET framework and its powerful decision-making tools. 


\begin{tabular}{|l|l|}
\hline LDRD\# 2018-093 & Securing Energy and Critical Resources \\
\hline Project Title: & $\begin{array}{l}\text { Real-Time Characterization of VTR Coolant Variables by High Resolution } \\
\text { Distributed Sensing }\end{array}$ \\
\hline Investigators: & $\begin{array}{l}\text { Darius Lisowski } \\
\text { Alexander Heifetz, Craig Gerardi, Hual-Te Chien, Sasan Bakhtiari }\end{array}$ \\
\hline
\end{tabular}

\section{Abstract}

Advanced instrumentation capabilities will enhance the utility of the Versatile Test Reactor (VTR) program and of VTR experiments seeking to provide key insights for advanced reactor performance. This project establishes experimental testing capabilities that provide critical insights into system behavior and flow phenomena within the reactor system. Specifically, characteristics such as flow near core regions and within thermal mixing tees are obtained with the high-fidelity data made available for computational validation. A suite of advanced sensors has been developed that provides real-time measurements of VTR thermal hydraulic characteristics with high resolution, high accuracy, and in-situ capability to monitor the near-core region, thermal mixing tees, experimental assemblies, and test loops. We demonstrated application of new sensors for measurements not accessible with current instrumentation. Fabrication of a test apparatus simulating a prototypic VTR environment was completed, along with characterization and performance evaluation of three sensors. Conversion of the test loop to liquid Galinstan (a surrogate for sodium) was also completed. Installed sensors include a distributed temperature sensor (DTS) across three two-meter long fibers, two ultrasonic Doppler velocimetry (UDV) probes, and a hermetically sealed notch wire transducer. The high-fidelity data obtained with the sensors can be used for detailed reactor performance assessment and to enhance reactor design using advanced computational tools. Our work will significantly enhance VTR experimentation, measurement, and data analysis capabilities. The in-core components and test assemblies are significant drivers in influencing VTR performance and operation. Their complex and unique thermal hydraulic behavior present significant design and modeling challenges that will be resolved by the new instrument capabilities. These instrumental capabilities will substantially elevate the impact of the VTR program on the design and operation of fast reactors and enable key insights into the physical behavior of advanced reactors.

\begin{tabular}{|l|l|}
\hline LDRD\# 2018-095 & Securing Energy and Critical Resources \\
\hline Project Title: & Towards the Creation of an Advanced Mobility Cybersecurity Testbed \\
\hline Investigators: & $\begin{array}{l}\text { Roland Varriale } \\
\text { Eric Rask, Nate Evans }\end{array}$ \\
\hline
\end{tabular}

\section{Abstract}

Argonne is currently researching and deploying infrastructure related to a wide variety of advanced mobility topics, including Vehicle to Infrastructure (V2I) and Vehicle to Vehicle (V2V) communications as well as Connected Automated Vehicles (CAVs), all of which will be able to interact in a controlled manner. By analyzing the interactions between these systems-more specifically the protocols and information exchanges as well as the system critical hardware itself (vehicles, sensors, Al aided decisions systems) - we can evaluate the security posture of a proposed advanced mobility concept and offer recommendations on potential weaknesses in both security and privacy. Creating an advanced mobility 
testbed will aid the analysis and production of security solutions and concepts across many transportation modes. As machine learning (ML)-based techniques become more widespread, research has begun to focus on techniques to respond to unintended or malicious inputs. This is especially important for vehicle technologies, given that ML has become an integral component of a vehicle's ability to detect and classify objects, ultimately becoming the "driver" in a fully automated vehicle. Additionally, ML-based analysis often underpins cybersecurity-related intrusion detection systems. However, ML-based systems are currently susceptible to adversarial network approaches. Therefore, robustness to adversarial approaches is a pressing need of more secure ML implementations. Much of this cybersecurity work can be done in a virtualized environment, but obtaining actual system responses, component data, communications data streams, and validation examples from vehiclesystem-sensor hardware is critical within the larger cybersecurity R\&D space. We are therefore designing and creating a specialized physical testbed to supplement efforts in virtual space. This testbed allows the collection and validation of cybersecurity-relevant operations within a flexible, safe, and repeatable laboratory space. We have created prototype software models and obtained physical hardware for verification of the models. Also created is a template control area network (CAN) simulation environment with data recording/playback and a configurable user environment supported by a relational database management system. This will allow us to prototype various CAN architectures and dynamically configure electronic control units (ECUs) by specifying a structured data file. On the laboratory development sub-project, a Man-in-the-Middle proof of concept has been developed using the Adaptive Cruise Control System (ACC) of a Toyota Prius. This capability allows researchers to override the information related between the vehicle's radar system and controls and thus can be used to impact how (if) a vehicle follows a lead vehicle when the vehicle's ACC system is active. Many unique possibilities are now available to understand how the vehicle reacts to unexpected sensor inputs as well as methods to better protect against these types of attacks. Lastly, we developed a bench-top laboratory for investigation of sensor susceptibility to outside attacks and "misinformation" via Generative Adversarial Network (GAN) techniques and related methods.

\section{THE UNIVERSE AS OUR LABORATORY (ULAB)}

\begin{tabular}{|l|l|}
\hline LDRD\# 2017-058 & The Universe as Our Laboratory (ULab) \\
\hline Project Title: & $\begin{array}{l}\text { A Strategic Scientific Program to Establish Argonne Leadership in the } \\
\text { Development of the Future Electron-Ion Collider }\end{array}$ \\
\hline Investigators: & $\begin{array}{l}\text { Kawtar Hafidi, Jose Repond } \\
\text { Adam Freese, Alexander Plastun, Brahim Mustapha, Cédric Mezrag, David Blyth, } \\
\text { David Potterveld, Geoffrey Bodwin, lan Cloet, Jessica Metcalfe, Jinlong Zhang, } \\
\text { John Arrington, Jose Martinez Marin, Jose Repond, Junqi Xie, } \\
\text { Mohammad Hattawy, Peter Ostroumov, Radja Boughezal, Sereres Johnston, } \\
\text { Sergei Chekanov, Stephen Magill, Thomas LeCompte, Whitney Armstrong, } \\
\text { Zachary Conway, Zhihong Ye }\end{array}$ \\
\hline
\end{tabular}

\section{Abstract}

An Electron-Ion Collider (EIC) has been identified in the Nuclear Science Advisory Committee's 2015 Long Range Plan for Nuclear Science as the highest priority for new facility construction in the United 
States. The EIC will have unique capabilities for colliding polarized electrons with polarized protons, polarized light ions, and heavy ions at high luminosity and with a versatile range of kinematics. These capabilities will allow unprecedented insights into the role of gluons, the carriers of the strong force, in building nucleons and nuclei and thus accounting for $98 \%$ of the mass in the visible universe. In support of the EIC, we pursued four tasks: theory, simulation, detector R\&D, and accelerator R\&D. Together, these efforts provide a detailed evaluation of the capabilities of the facility and a path to optimizing the final results. Our goal in the theory task was to calculate observables (e.g., cross sections, angular distributions) that can be measured at the EIC and that directly probe gluonic content. The outcome of the theory effort is to guide the design and experimental program of the EIC. Under this task, we developed the first relativistic and chiral effective field theory that respects the symmetries of quantum chromodynamics $(Q C D)$ to calculate the generalized parton and transverse momentum distribution functions of the deuteron. Our simulation work focused on the essential physics processes expected at the EIC through full simulation of detector responses. We proposed a new physics-optimized detector for the EIC, which aided in identifying new technologies for the R\&D tasks of this project. In the detector R\&D task, we developed a novel 5D detector featuring high-granularity spatial, timing, and energy measurements. We developed a novel state-of-the-art detector concept for the EIC named TOPSIDE and assembled a complete simulation and reconstruction tool chain for it. We also started an effort to develop ultra-fast silicon detectors. Finally, in the accelerator R\&D task, we completed a new alternative design for the EIC's ion accelerator complex, including the design of a new ion pre-booster, based on a compact linac and an already-designed octagonal 3-GeV pre-booster with room-temperature magnets.

\begin{tabular}{|l|l|}
\hline LDRD\# 2018-012 & The Universe as Our Laboratory (ULab) \\
\hline Project Title: & $\begin{array}{l}\text { A High Precision Superconducting Detector for Next Generation Neutrinoless } \\
\text { Double Beta Decay Search Experiments }\end{array}$ \\
\hline Investigators: & $\begin{array}{l}\text { Gensheng Wang } \\
\text { Clarence Chang, Junjia Ding, Valentine Novosad, Volodymyr Yefremenko, } \\
\text { Whitney Armstrong }\end{array}$ \\
\hline
\end{tabular}

\section{Abstract}

Determining the existence of neutrinoless double beta decay (NLDBD) is among the highest priorities in neutrino physics. NLDBD is a decay mode of certain nuclei where two neutrons convert to two protons and two electrons with no emission of neutrinos. A positive observation of NLDBD, the search for which is the favored technique for exploring the neutrino's possible Majorana nature, would conclusively show that neutrinos are so-called Majorana particles, profoundly impacting our understanding of fundamental physics as NLDBD decay is allowed only if neutrinos are Majorana particles. The search for NLDBD is challenging because the decay is rare (half-life greater than 1025 years) requiring that experiments have target masses on the order of one ton and operate with very low radioactive backgrounds (less than 0.1 counts/ton/year/keV). It is critical for NLDBD experiments to develop detector technology that is both scalable in mass and capable of active background rejection. Building on our expertise with superconducting transition edge sensors (TESs) in the study of cosmic microwave background (CMB) radiation, we are developing a TES bolometric detector with the potential for both scalability and background discrimination for a large-scale NLDBD search experiment. Our project provides a unique TES detector technology utilizing a low superconducting transition temperature (low-Tc) for applications 
that require low-threshold and high resolution. The low threshold and high energy-resolution of the TES will enable measurements of multiple decay signals (e.g., Cherenkov emission and heat, or scintillation and heat) which enable particle identification and thus background rejection. We have developed two reproducible fabrication recipes for a low-Tc TES. We set up a cryogenic testing system with multiple sensitive SQUID electronics readout channels for characterizing the properties of a low-TC TES detector. This system provides high precision resistance versus temperature measurements for characterizing the superconducting-to-normal transitions for our thin films. We have measured the transition temperatures (Tc) for a series of samples and detectors including: sputtered films and patterned devices made of iridium/platinum (Ir/Pt) bilayers, as well as gold-iridium ( $\mathrm{Au} / \mathrm{Ir} / \mathrm{Au}$ ) trilayer samples.

\section{DIRECTOR'S COLLABORATIONS}

\begin{tabular}{|l|l|}
\hline LDRD\# 2017-156 & Director's Collaborations \\
\hline Project Title: & Scalable Data Movement for Data-centric Supercomputing \\
\hline Investigators: & Venkatram Vishwanath \\
\hline
\end{tabular}

\section{Abstract}

Understanding, characterizing, and optimizing application data flows on next generation supercomputer architectures is difficult. The difficulty lies in effectively abstracting the system architecture; mapping the complex organization of the system architecture with the data movement requirements in both space and time, such that the information can be used to explore trade-offs (such as power consumption versus execution time); and implementing transformations and mapping that may result in better performance. Moreover, these supercomputing infrastructures are being shared by diverse, concurrent applications, including those that require data-intensive flows. New approaches are required for taking us to the next level in understanding interactions between system infrastructure and application data flows at extreme scales. Our project will help accelerate the transformation of raw data into insights and discoveries and improve our understanding of the data-centric infrastructures needed at exascale and future facilities. We have defined resource abstraction models, taking into account complex supercomputing architectures expected to be deployed at Argonne. Our initial focus was on network topology. We have designed algorithms for data movement optimizations accounting for system topology. In one case, we were able to improve the data movement for a data-intensive cosmology application kernel by $45 \%$ on the Mira Blue Gene/Q supercomputer.

\begin{tabular}{|l|l|}
\hline LDRD\# 2018-106 & Director's Collaborations \\
\hline Project Title: & Synergistic Multi-Site Catalysts For Dry Reforming Processes \\
\hline Investigators: & $\begin{array}{l}\text { Massimiliano Delferro } \\
\text { Cong Liu, Maria Chan }\end{array}$ \\
\hline
\end{tabular}

\section{Abstract}

Catalytic conversion of carbon dioxide to other chemicals has been investigated as an approach to mitigate global carbon dioxide $\left(\mathrm{CO}_{2}\right)$ emissions. The focus of this project is on $\mathrm{CO}_{2}$-assisted natural gas 
reforming, referred to as dry reforming of methane (DRM), which can be integrated with processes for fuel and chemical production. DRM converts two greenhouse gases $\left(\mathrm{CO}_{2}\right.$ and $\left.\mathrm{CH}_{4}\right)$ into syngas $(\mathrm{CO}$ and $\mathrm{H}_{2}$ ), which can be transformed into value-added chemicals and fuels by methanol or Fischer-Tropsch synthesis reactions. The key to our approach is to design multi-site catalysts, where each step of the complex process occurs on a neighboring surface site that not only possesses the required activity and selectivity toward the specific reaction step of the process, but is also able to act in concert with its neighbors to complete the catalytic reaction. Our main focus is on the combination of multimetallic active sites over highly reducible oxides. By means of high-throughput synthesis and testing facilities at Argonne, we've discovered bimetallic catalysts supported on different metal oxides. Specifically, we synthesized nickel-containing and platinum-containing bimetallic catalysts with different secondary metals (boron, zinc, manganese, cobalt, vanadium, copper, iron, magnesium, and tin) over six different metal oxide supports (silica, alumina, titania, ceria, strontium titanate, and magnesium oxide). The resulting catalysts were tested for dry reforming of methane and $\mathrm{CO}_{2}$ methanation. Achieving high stability and activity was very dependent on the catalyst support and bimetallic nature. The most promising catalyst formulations have been identified. Boron, when used as a promoter, facilitates the migration of carbon groups from the active Pt sites, and thus enhances the catalytic stability. Our X-ray photoelectron and nuclear magnetic resonance spectroscopy investigations over fresh and used catalysts revealed the effect of promoter metal in extending the lifetime of the catalysts. The experimental work was augmented and guided by first principles quantum mechanical calculations and application of machine learning techniques to correlate observed catalytic activity with synthesis protocols.

\begin{tabular}{|l|l|}
\hline LDRD\# 2018-107 & Director's Collaborations \\
\hline Project Title: & $\begin{array}{l}\text { Creating Next-Generation Quantum Electronics with Topological } \\
\text { Insulator/Ferromagnetic Insulator Heterostructures }\end{array}$ \\
\hline Investigators: & $\begin{array}{l}\text { John Freeland } \\
\text { Anand Bhattacharya }\end{array}$ \\
\hline
\end{tabular}

\section{Abstract}

The investigation of topology and symmetry has led to the discovery of a new class of materials, known as topological insulators (TI), which host metallic surface states with behavior that can only be understood in the context of quantum mechanics. By influencing the surface carriers in Tls using a ferromagnetic insulator (FMI) layer placed in proximity at the atomic level using epitaxial growth, we could harness these quantum phenomena to provide enabling technology for next-generation magnetic random-access memory, logic devices, dissipation-less interconnectors, and quantum bits for computation. However, we first need to understand the underlying physics of how topologically protected surface states couple to magnetism at the atomic level. Two critical questions are addressed in this project: (1) What are the optimum structures or materials for TI/FMI heterostructures to switch their magnetization? And (2), is it possible to develop room-temperature magnetic TIs? Our experimental effort can pave the way to understanding the critical role of interface properties in the quantum phenomena of topological phases. We have fabricated films of bismuth/antimony (Bi/Sb) and of $\mathrm{Bi}$ itself (in a 111 orientation) on silicon ( $\mathrm{Si}$ ) and gallium arsenide (GaAs) substates. Both films are believed to show TI behavior. Magnetic insulator substrates included yttrium iron garnet (YIG). We have 
observed the planar Hall effect and anisotropic magnetoresistance in our films. We tracked the behavior via simultaneous time resolved soft X-ray measurement of the magnetism and in-situ transport measurements.

\begin{tabular}{|l|l|}
\hline LDRD\# 2018-116 & Director's Collaborations \\
\hline Project Title: & Long-Lived Rare-Earth Qubits on Silicon \\
\hline Investigators: & Supratik Guha \\
\hline
\end{tabular}

\section{Abstract}

The development of individually addressable rare-earth quantum bits (qubits) remains unfulfilled and represents the frontier of quantum information science. We are developing a solid-state qubit platform based on epitaxial yttrium oxide $\left(\mathrm{Y}_{2} \mathrm{O}_{3}\right)$ thin films doped with erbium ions, deposited using molecular beam epitaxy (MBE), and sandwiched between silicon layers to make a heterostructure nanophotonic waveguide. This scalable quantum technology combines long coherence with a powerful silicon nanophotonic architecture to enable high-performance qubits and quantum computing devices. Rareearth (RE) ions in solids are promising candidates, as they feature $4 \mathrm{f}$ intra-shell transitions that are shielded from surroundings by closed outer shells, making the ions immune to noise and imperfections in the crystal lattice. This affords ultra-long qubit coherence times and narrow optical transitions. The erbium ion was chosen because it has optical transitions at the telecom wavelength of 1,535 nanometers $(\mathrm{nm})$, which is compatible with existing fiber-optic telecommunication infrastructures. Meanwhile, the isotope erbium-167 $\left({ }^{167} \mathrm{Er}\right)$ is already demonstrated to have long nuclear spin coherence times exceeding seconds. We performed high resolution coherent spectroscopy on the synthesized materials to extract qubit coherence times in order to verify long-lived qubits. Another unique advantage of this approach is that it enables efficient routing of quantum signals for qubit control. Realization of long-lived, fully controllable qubits is a central theme in quantum information technology, which is poised to revolutionize computing, data analytics, materials science, and pharmaceutical research.

\begin{tabular}{|l|l|}
\hline LDRD\# 2018-117 & Director's Collaborations \\
\hline Project Title: & $\begin{array}{l}\text { Dynamic Cross-linked Polymers for Silicon-based Electrodes in Lithium-Ion } \\
\text { Batteries }\end{array}$ \\
\hline Investigators: & Daniel Abraham \\
\hline
\end{tabular}

\section{Abstract}

Although current lithium-ion batteries typically use graphite anodes, silicon-based anodes have promised higher capacities for next-generation lithium-ion batteries. However, when lithium ions are inserted and removed from silicon, a large volume change occurs. This causes mechanical failure of the anodes and reduced battery lifetime. Also, a so-called solid electrolyte interphase forms on the silicon surface which cracks and breaks off, exposing the silicon surface to a battery's electrolyte, and more interphase formation. One key to addressing both problems is a polymer binder that serves to "glue" all contents of the anode together. As large volume changes are intrinsic to the silicon particles, the 
artificial solid electrolyte interphase needs to be an elastic material (elastomer) that can be stretched without fracturing during stress while still permitting lithium-ion transport. In this project, we are developing and characterizing a binder material that will enable silicon-based anodes for nextgeneration lithium-ion batteries. Conductive dynamic crosslinked polymers can serve as binder in these silicon electrodes. We measured the mechanical strength and adhesive properties of the polymer networks. We determined the lithium-ion conductivities of the polymers in the room-temperature to $180^{\circ} \mathrm{C}$ range, and we incorporated these polymers into a mixture of silicon and carbon nanoparticles, fabricated battery electrodes and tested them under battery-relevant conditions.

\begin{tabular}{|l|l|}
\hline LDRD\# 2019-0383 & Director's Collaborations \\
\hline Project Title: & Sustainability of Brackish Groundwater in the Mid-Continental Region \\
\hline Investigators: & $\begin{array}{l}\text { Peter Mueller } \\
\text { Jake Zappala }\end{array}$ \\
\hline
\end{tabular}

\section{Abstract}

Population growth and climate change are primary causes of increasing water stress. Yet brackish groundwater remains a widely available as a non-traditional water resource. Combined with rapidly improving desalination technology, it may provide an adequate and affordable water supply. However, the sustainability of water resources cannot be determined by simple volume estimation of the water reservoirs alone; also required is knowledge of their dynamic properties, such as aquifer connectivity and effective flow velocity. Subsurface residence time of groundwater provides crucial information on the dynamic properties of hydrological systems. Groundwater tends to mineralize (gain dissolved solids) as it ages. Accordingly, brackish mid-continental groundwater (i.e., not from current seawater intrusion) most probably has spent a substantial length of time in the subsurface. The absence of a groundwater age tracer on timescales greater than 50,000 years makes it difficult to assess the resource potential of brackish water reservoirs. Our project seeks to provide such an assessment by exploiting a tracer of old groundwater age, Krypton-81 ( ${ }^{81} \mathrm{Kr}$, half-life of 229,000 years) This is available at Argonne's Trace Radioisotope Analysis Center using the Atom Trap Trace Analysis technique. Ultimately, we hope to determine, in fine geographic detail, the sustainable yields of brackish groundwater from the Northern Great Plains aquifer system and the Midwest states of the Ohio River Valley where recent studies suggest that together they have significant brackish water reservoirs, and that both have high agricultural and industrial water demands. We have identified reservoirs in the Ogallala aquifer for initial evaluation and have collected available data on the region. 


\section{NAMED FELLOWS}

\begin{tabular}{|l|l|}
\hline LDRD\# 2016-183 & Named Fellow \\
\hline Project Title: & $\begin{array}{l}\text { Understanding and Controlling Charge, Spin, Pseudospins and Lattice Degrees } \\
\text { of Freedom in Layered Transition Metal Dichalcogenides }\end{array}$ \\
\hline Investigators: & $\begin{array}{l}\text { Qi Zhang } \\
\text { Haidan Wen }\end{array}$ \\
\hline
\end{tabular}

\section{Abstract}

The goals of this project are to understand and control the dynamics of charge, spin, and pseudospins by low-energy excitation; to explore strong field physics in layered transition metal dichalcogenides (TMDs) with ultrafast terahertz ( $\mathrm{THz}$ ) pump-probe spectroscopy; and to use a laser pump and a hard X-ray nanodiffraction probe at Argonne's Advanced Photon Source (APS) and the Linear Coherent Light Source (LCLS) to study ultrafast lattice dynamics of laser-induced phase transitions in TMDs. Twodimensional (2D) layered materials -- particularly the TMDs -- display coupled quantum degrees of freedom, including spin, and valley and layer pseudospins, with electrical and optical accessibility. These could be the basis for new-concept advanced optoelectronics devices that offer high information density, low energy cost, and precise timing. We must first understand the fundamental dynamics of the electronic degrees of freedom under optical excitation. We built two THz spectroscopy systems and also conducted a successful experiment using the ultrafast X-ray diffraction technique. THz system is the world's first cryogenic near-field THz spectroscopy system. Unique structural dynamics of monolayer crystals and pressure tuning 2D magnetic states were studied. Ultrafast lattice dynamics in TMDs was revealed by ultrafast X-ray diffraction at the LCLS. We discovered a surprisingly fast (5 ps) lateral lattice expansion in monolayer WS $\mathrm{S}_{2}$ flakes after intense optical excitation. We also demonstrated that a femtosecond spin current pulse can generate THz transients at Rashba interfaces between two non-magnetic materials $(\mathrm{Ag} / \mathrm{Bi})$. Our result is the first ultrafast demonstration of the inverse Edelstein effect. We have recently discovered strongly coupled lattice dynamics and magnetic dynamics in novel intrinsic layered magnets $\mathrm{Crl}_{3}$. Lastly, We achieved gate-tunable $\mathrm{THz}$ emission at the interface between $\mathrm{LaAlO}_{3} / \mathrm{SrTiO}_{3}$, based on interfacial photoinduced spin-to-charge conversion

\begin{tabular}{|l|l|}
\hline LDRD\# 2017-031 & Named Fellow \\
\hline Project Title: & $\begin{array}{l}\text { Ecological Organic Photovoltaics using Water-borne Semiconductor } \\
\text { Nanoparticles }\end{array}$ \\
\hline Investigators: & $\begin{array}{l}\text { Yu Jin Kim } \\
\text { Gary Wiederrecht, Seth Darling }\end{array}$ \\
\hline
\end{tabular}

\section{Abstract}

This project resulted in the synthesis of water-processable $\mathrm{n}$ - and $\mathrm{p}$-type semiconducting colloidal particles that could assemble into the photoactive layer of extremely efficient photovoltaic devices. It also led to the synthesis of n-type nanospheres with embedded quantum dots with unique optical properties that were characterized by fluorescence resonance energy transfer. Organic photovoltaics have promise as the next-generation renewable energy source with their light weight, mechanical flexibility, and low-energy manufacturing. However, harmful chemicals, particularly chlorinated solvents, 
are required for their manufacture. The accomplishment of this project has been development of an environmentally friendly alternative solvent-specifically water-to replace chlorinated solvents in organic photovoltaic manufacturing. The key to enabling the dispersal of active organic materials in a water solution is to use surfactants to develop water-borne colloids/nanoparticles of organic semiconductors. Water-borne organic semiconductor nanoparticles with a precisely separated heterojunction structure have been successfully synthesized and applied to our ecological photovoltaic devices. Moreover, in-situ grazing-incidence wide-angle X-ray scattering during spin coating probed the deposition of the $n$ - and $p$-type colloidal particles from aqueous solutions and revealed the morphological transformations of the particles into thin film materials. The new structure of the particle with its unexpected photonic property resulted in world-class device performance.

\begin{tabular}{|l|l|}
\hline LDRD\# 2017-094 & Named Fellow \\
\hline Project Title: & Microstructural Simulations of Stable Conjugated Polymer Glasses \\
\hline Investigators: & $\begin{array}{l}\text { Nicholas Jackson } \\
\text { Juan De Pablo, Venkatram Vishwanath }\end{array}$ \\
\hline
\end{tabular}

\begin{abstract}
Organic electronics underpin a variety of potentially revolutionary technologies, including inexpensive solar energy devices, flexible transistors, and biological interfaces. Two fundamental deficiencies have kept them from meeting cost and performance requirements for widespread industrial adoption: i.e., lack of rational control of microstructural morphology of organic semiconductors and susceptibility of metastable, kinetically trapped, organic films to thermal and photo-induced degradation. Precise microstructural control offered by physical vapor deposition (PVD) makes it a potential processing path for mitigating deficiencies via increased device lifetimes and performance by design. This project sought computational techniques, for analysis of structural and charge transport properties of glassy polymeric materials, that will aid that design. We have implemented an "Electronic Coarse Graining" methodology that utilizes machine learning to accelerate optoelectronic predictions in soft materials. We've established structure property relations for glass polyelectrolyte brushes in the presence of multivalent ions. And, we developed an advanced sampling scheme (Layered Nested Markov Chain Monte Carlo) for improved sampling in systems with slow dynamics. The simulation and visualization tools developed here will strongly support application to PVD on an experimentally relevant scale. They will allow exploration of polymer morphologies obtainable by systematic variations in polymer structure and PVD conditions. And, they will aid in characterizing the influence of these variables on a film's optoelectronic functionality and susceptibility to thermal and photo-induced degradation.
\end{abstract}




\begin{tabular}{|l|l|}
\hline LDRD\# 2017-097 & Named Fellow \\
\hline Project Title: & A Universal Data Analytics Platform for Science \\
\hline Investigators: & $\begin{array}{l}\text { Ryan Chard } \\
\text { lan Foster }\end{array}$ \\
\hline
\end{tabular}

\section{Abstract}

Scientific discovery is increasingly reliant on big data, sophisticated data analytics, and large-scale computing infrastructure. Exponential growth of data volumes, increasing data heterogeneity, and exploding analytical complexity drives the need for efficiently and reliably managing, curating, synthesizing, and analyzing data. This is a challenge faced in almost every scientific domain. To address the challenges associated with managing and analyzing large amounts of data, we have researched and developed an extensible data-oriented analytics platform, essentially a web-based universal dataoriented analytics platform. The platform makes scientific data, regardless of repository location, readily accessible and manageable, enables analysis of disparate data using cutting edge analytics tools, and transparently employs autonomic computing methodologies to optimize the execution of analytics tools on a broad set of large-scale computing infrastructures. This project enables a wide variety of distributed data to be more easily integrated and analyzed, thus supporting larger and more complex scientific inquiries. It democratizes access to cutting-edge and best-practices approaches and to largescale cyber infrastructure. A prototype responsive storage implementation, called "Ripple", has been implemented and deployed to automate a set of the identified use cases. This prototype is currently deployed on a range of computing environments, including resources at NERSC, LBL, and the APS, and is able to automate analysis pipelines for light source science.

\begin{tabular}{|l|l|}
\hline LDRD\# 2018-111 & Named Fellow \\
\hline Project Title: & Bio-inspired Smart Soft-Robots \\
\hline Investigators: & $\begin{array}{l}\text { Xu Zhang } \\
\text { Daniel Lopez }\end{array}$ \\
\hline
\end{tabular}

\section{Abstract}

Deformable biological systems show a great variety of forms and functionalities. The field of "soft robots" takes its inspiration from these biological systems. Soft robots made from deformable materials can adapt to changing environments and tasks. For example, soft robots promise to be particularly capable of locomotion over unknown terrain for search-and-rescue applications. Existing soft robots still have limited abilities to sense, compute, and communicate. Wirelessly powering soft robots is another challenge. An obstacle is their flexibility which precludes use of conventional rigid sensors, communication circuits, and power sources. Atomically thin and mechanically robust two-dimensional (2D) materials are suited for embedding intelligence into soft robots. We aimed to integrate 2D-materials-based soft electronics into soft robots to create "smart soft robots" with smart skin and reconfigurable and programmable bodies. Smart skin includes large-area flexible multi-pixel pressure sensor arrays for "touch reception." Energy harvesting, signal conditioning, and wireless communication can also be integrated into the smart skin. Our goal is to build an untethered reconfigurable soft robot. Graphene, semiconducting transition metal dichalcogenides, and carbon nanotubes (CNT) are used for soft electronics. Unlike the conventional "electronic intelligence" in computers and conventional robots, 
the soft robots arguably exhibit "mechanical intelligence", in which the boundary between the brain and body becomes blurred. The soft bodies can augment the brain with morphological reconfigurability and also simplify the control algorithms. The "smart skin" incorporates an on-board building block of signal conditioning based on $\mathrm{MoS}_{2}$ to allow on-site data processing. In addition, a MoS 2 -based flexible rectifying antenna is used as an RF wireless charger. A flexible antenna based on highly aligned carbon nanotubes (CNTs) is fabricated for both the $\mathrm{MoS}_{2}$ rectifying antenna and the wireless communication. Metalenses (flat surfaces that use nanostructures to focus light) have been applied to silicon nitride membrane substrates and Kirigami structures have been fabricated using a focused ion beam and actuated using Joule heating.

\begin{tabular}{|l|l|}
\hline LDRD\# 2018-112 & Named Fellow \\
\hline Project Title: & Engineered Materials for Additive Manufacturing \\
\hline Investigators: & $\begin{array}{l}\text { Sarah Wolff } \\
\text { Aaron Greco, Tao Sun }\end{array}$ \\
\hline
\end{tabular}

\section{Abstract}

Additive manufacturing (AM) is capable of making multi-material and functional components with complex geometries through rapid and localized directional solidification of molten metallic alloys derived from micro-scale powder precursors. We investigated the physics underlying directed energy deposition (DED), a powder-blown AM process, and the engineering of new materials. The DED process can build complex geometries with multiple materials at multiple spatial resolutions and superior mechanical properties may be achieved. Our project addressed the engineering new materials for AM by combining computational efforts with in-situ X-ray imaging and diffraction studies during the AM process. Monitoring and imaging of the processes opens many possibilities for materials design of new functionally graded alloys. We have measured the thermal histories, in particular cooling behavior, as part of in-situ melt pool characterization and shown how it can lead to prediction of porosity. Results have been input to machine learning algorithms. We performed an in-situ study of laser powder bed fusion using simultaneous high speed infra-red and X-ray imaging to detect porosity. We found that powder flow drastically influences laser attenuation into the melt pool and that powder can melt differently depending on laser parameters. We observed that powder flow can cause porosity and that it is capable of building dissimilar materials. 
INNOVATE

\begin{tabular}{|l|l|}
\hline LDRD\# 2017-019 & Innovate \\
\hline Project Title: & $\begin{array}{l}\text { Bonding Dissimilar Materials using Nanoparticles/Nanofilm as Eutectic } \\
\text { Compounds }\end{array}$ \\
\hline Investigators: & $\begin{array}{l}\text { Kamleshkumar Suthar } \\
\text { Harry Fry, Lahsen Assoufid, XianRong Huang }\end{array}$ \\
\hline
\end{tabular}

\section{Abstract}

Primary components for accelerators are fabricated by bonding dissimilar materials such as non-metallic materials with low coefficients of thermal expansion (CTEs) (quartz and silicon) and metals, which have high CTEs. Current bonding techniques produce unacceptable strain, induce heat damage, or create devices that cannot be operated at high temperatures. Our project is developing a virtually stress-free bonding technique using low-melting-temperature nanoparticles and nanofilms of reactive metals. The new technique takes advantage of two distinctive characteristics of nanomaterials: (1) they melt at much lower temperatures than the corresponding bulk materials and (2) they react aggressively to generate localized high temperatures. Our plan included bonding at low temperatures using lowmelting-point nanoparticles or by locally creating high temperatures using reactive nano-thermite materials. The resulting bonds should be thermally efficient, be structurally sound, and leave minimal residual thermal stresses in the bulk material. The assimilated nanoscale materials will assume properties of the bulk material, including its higher melting temperature. Further, the nanomaterials can be configured (e.g., placed in alternating layers) to modulate the amount of heat released into the bulk, thus minimizing recrystallization and annealing during bonding. We successfully bonded copper pieces to a Si monochromator and produced and tested a strain-free X-ray intercepting surface. We synthesized 4-nm size titanium (Ti) nanoparticles, investigated their reactivity, and imaged them in a TEM. Finally, we have produced ultra-thin $100-\mathrm{nm}$ gold $(\mathrm{Au})$ nanoporous foil for incorporation at the interface during bonding to increase strength and thermal conduction.

\begin{tabular}{|l|l|}
\hline LDRD\# 2017-026 & Innovate \\
\hline Project Title: & Realizing a Gate Tunable Kinetic Inductance for a Transmon Qubit using $\mathrm{SrTiO}_{3}$ \\
\hline Investigators: & $\begin{array}{l}\text { Anand Bhattacharya } \\
\text { Alex Martinson, Jidong Samuel Jiang }\end{array}$ \\
\hline
\end{tabular}

\section{Abstract}

Superconducting circuits incorporating Josephson junctions (JJs) have emerged as one of the most promising avenues for realizing qubits in the solid state, with coherence times approaching the threshold needed for fault-tolerant quantum computing. A transmon is a combination of a JJ and a capacitor, which form elements of a nonlinear (anharmonic) oscillator. A particular qubit can be addressed or coupled to another qubit by tuning the inductance of the JJ circuit. Conventional transmons are susceptible to stray magnetic fields and are difficult to miniaturize. The central idea of this project is to create an electric-field-gated superconducting channel by using a low-carrier-density superconductor, $\mathrm{SrTiO}_{3-\delta}$ (n-STO), and low carrier density semimetals such as $\mathrm{Bi}, \mathrm{Sb}$ and $\mathrm{YPdBi}$, and to tune the kinetic inductance of the resulting device via gate voltage. STO doped with oxygen vacancies 
becomes superconducting at extremely low carrier densities, which provides for quite tunable gates in a thin film or at interfaces using conventional dielectrics, such as $\mathrm{HfO}_{2}$. In addition, the kinetic inductance of a gated n-STO superconducting channel, which scales inversely as the superfluid density (and thus also the critical current), will be very large and tunable over a large range of values. Reducing the channel length to nanoscale dimensions $(10-500 \mathrm{~nm})$ will be the key to realizing a robust and gatetunable super current in our n-STO based devices. We use He-ion focused ion beam (FIB) etching and e-beam lithography to accomplish that.

\begin{tabular}{|l|l|}
\hline LDRD\# 2019-0006 & Innovate \\
\hline Project Title: & Deep Learning for Posterior Density Emulation and Sampling \\
\hline Investigators: & $\begin{array}{l}\text { Carlo Graziani } \\
\text { Julie Bessac }\end{array}$ \\
\hline
\end{tabular}

\section{Abstract}

Scientists often need to compare experimental data with a family of scientific models to determine which model or models are more likely to be correct. The state-of-the-art Bayesian approach to this question, called "Markov Chain Monte Carlo" (MCMC) is often difficult to implement efficiently. We have created an alternative approach to this model-data comparison activity. The new method is based on deep learning techniques, and may remedy some difficulties attendant to the MCMC method. The new type of deep learning network is adapted to the task of emulating general Bayesian posterior densities. Our method can be more efficient than MCMC for extracting sampled information from such densities. We demonstrated that inference with complex models is possible using the new method and that the results do compare favorably to MCMC-based results. Such a new inference method constitutes an addition to the current arsenal of uncertainty quantification tools.

\begin{tabular}{|l|l|}
\hline LDRD\# 2019-0010 & Innovate \\
\hline Project Title: & Large-Scale Uncertainty Quantification for Power System Resilience \\
\hline Investigators: & $\begin{array}{l}\text { Michel Schanen } \\
\text { Daniel Maldonado }\end{array}$ \\
\hline
\end{tabular}

\section{Abstract}

Weather-dependent energy sources expose the grid to extreme events. To properly determine the measures needed for improving the electrical grid's resilience, the uncertainties of such events and operating conditions must be quantified and included in planning policies. Stability studies for power systems entail the integration of a set of differential algebraic equations, which are perturbed to represent a grid fault, and a set of criteria to determine if the regime after the perturbation is safe and stable. Distributed energy resources bring epistemic uncertainty which needs to be modeled as a probability distribution function in the initial conditions or parameters. Uncertainty quantification (UQ) in power system stability studies involves the propagation of uncertain initial conditions and parameters through the governing non-linear equations. The dimension of typical electrical power systems is such that standard UQ methods are unable to scale. We have developed a scalable methodology to enable 
UQ in power system stability simulations. Our UQ methodology leverages automatic differentiation to propagate statistical descriptors through the nonlinear dynamics. The current implementation allows us to accurately propagate uncertainty in systems of up to 1,000 electrical buses. Our ultimate goal is to break the barrier toward 10,000 buses, showcasing that our method of assessing uncertainty is applicable to realistic grid sizes in the United States. Our software package is able to compute the uncertainty using the method of moments at an unprecedented scale and speed. This research improves the resilience of the electrical grid by reducing reliance on heuristics for reliability assessment. At present, no other methodology exists that can effectively tackle the UQ in high-dimensional problems that arise in the analysis of energy infrastructures.

\begin{tabular}{|l|l|}
\hline LDRD\# 2019-0024 & Innovate \\
\hline Project Title: & Krypton-85 Analysis in Uranium Hexafluoride via Atom Trap Trace Analysis \\
\hline Investigators: & $\begin{array}{l}\text { Peter Mueller } \\
\text { Derek McLain, Jake Zappala }\end{array}$ \\
\hline
\end{tabular}

\section{Abstract}

Krypton- $85\left({ }^{85} \mathrm{Kr}\right)$ is a noble gas radioisotope that is produced with high yield in the spontaneous and neutron-induced fission of uranium. Due to its long half-life (10.76 years), it accumulates in containers of uranium hexafluoride $\left(\mathrm{UF}_{6}\right)$ and can serve as a tracer of a sample's age and history for safeguard and verification purposes. We established atom trap trace analysis (ATTA) of ${ }^{85} \mathrm{Kr}$ in headspace samples extracted from $\mathrm{UF}_{6}$ bulk material storage containers. ATTA now offers highly selective and sensitive detection of ${ }^{85} \mathrm{Kr}$. Our method adds valuable information on the age and history of UF 6 samples and complements conventional radiochronometry techniques, especially for relatively "young" samples. We have completed: analysis of the ${ }^{85} \mathrm{Kr}$ production mechanisms in $\mathrm{UF}_{6}$ and specified required detection limits and precision for head-space analysis; development of a measurement protocol for ATTA analysis of head-space samples that can achieve a detection limit for ${ }^{85} \mathrm{Kr}$ below the 5000 -atom level; and obtained and analyzed ${ }^{85} \mathrm{Kr}$ calibration samples as well as actual $\mathrm{UF}_{6}$ head-space samples using this newly developed protocol. Solubility of $\mathrm{Kr}_{\text {in }} \mathrm{UF}_{6}$ is also measured. Beyond the initial goal of the project, we have identified a procedure to further reduce the ${ }^{85} \mathrm{Kr}$ detection limit of ATTA by an order of magnitude through laser-induced optical pumping. The accuracy of the method for samples of various ages, optimized sample extraction and handling, and ATTA procedures have also been addressed.

\begin{tabular}{|l|l|}
\hline LDRD\# 2019-0048 & Innovate \\
\hline Project Title: & A New View on Charge and Spin in Functional Metal Oxides \\
\hline Investigators: & Alexander Rettie \\
\hline
\end{tabular}

\section{Abstract}

We investigated how charge interacts with spin in transition metal oxides, pointing the way to improved and new metal oxide-based devices. Transition metal oxides are central to the production of solar fuels, emerging photovoltaics, energy storage, and catalysis. Breakthroughs in our understanding of these functional materials will enable improvements in the efficiency, stability, and cost of these technologies. 
A critical aspect of all such materials is their ability to carry charge. Numerous functional metal oxides exhibit pair-trapped charge carriers and magnetic order at room temperature, with the latter being thought to induce a barrier to electronic transport. Previous experiments on the iron oxide, hematite $(\alpha-$ $\mathrm{Fe}_{2} \mathrm{O}_{3}$ ), suggest that certain dopants may allow modulation of this barrier. We grew single crystals of hematite by the high-pressure float-zone technique and characterized their structure via X-ray diffraction to confirm crystal quality and purity. We also crystallographically aligned the samples in anticipation of making measurements of anisotropic conductivity. In addition to pure samples, we doped samples with silicon during growth (nominally $1 \%$ and $5 \%$ levels based on substitution for iron atoms) This produced electrically conductive $\mathrm{Si}: \alpha-\mathrm{Fe}_{2} \mathrm{O}_{3}$ single crystals. Measurements of the electrical conductivity anisotropy (in-plane vs. out-of-plane) for undoped and $\mathrm{Si}$-doped $\alpha-\mathrm{Fe}_{2} \mathrm{O}_{3}$ single crystals as a function of temperature were made. These measurements should lead to an understanding of how charge interacts with spin in transition metal oxides. Our intent is, provided tunable conductivity anisotropy is robustly shown in the doped $\alpha-\mathrm{Fe}_{2} \mathrm{O}_{3}$, to expand to other functional metal oxides with magnetic order (e.g., $\mathrm{NiO}, \mathrm{LaFeO}_{3}$ ) in order to demonstrate broad applicability of the phenomenon.

\begin{tabular}{|l|l|}
\hline LDRD\# 2019-0057 & Innovate \\
\hline Project Title: & $\begin{array}{l}\text { Development of an Integrated, Mechanistic Framework for Lead-Cooled Fast } \\
\text { Reactor Source Term Assessments }\end{array}$ \\
\hline Investigators: & $\begin{array}{l}\text { Matthew Bucknor } \\
\text { James Jerden, Acacia Brunett }\end{array}$ \\
\hline
\end{tabular}

\section{Abstract}

We seek to advance the state of the art of non-light water reactor safety analyses by developing an integrated software toolkit for mechanistic source term (MST) analyses that utilizes a consistent, accepted methodology. Source terms are the possible releases of radioactive material during reactor transients. A barrier to commercialization of advanced nuclear reactors is the lack of an accepted methodology for MST assessment. Beyond development of the toolkit itself, another goal is to increase the capabilities our software tools to enable MST assessments of lead-cooled reactors. Historically, these tools have been primary utilized for sodium-cooled reactor assessments. The initial stage of the project focused on review of existing literature and experimental data to develop temperature- and radionuclide group-dependent fission product release fractions. An assessment of fission product release and transport phenomena in lead-cooled reactors was then completed, a gap assessment of existing MST software tools was conducted for applicability to lead-cooled reactors, software gap resolutions were performed, and an integrated software toolkit, including a graphical user interface, was developed to perform MST assessments. Testing of the software toolkit with existing MST computational codes was conducted and a user's manual for the toolkit was developed. 


\begin{tabular}{|l|l|}
\hline LDRD\# 2019-0083 & Innovate \\
\hline Project Title: & $\begin{array}{l}\text { Production of Chemically and Mineralogically Accurate Far-Field Fall Out } \\
\text { Particles Using an Electric Arc Furnace }\end{array}$ \\
\hline Investigators: & $\begin{array}{l}\text { Derek McLain } \\
\text { David Chamberlain, Michael Kaminski }\end{array}$ \\
\hline
\end{tabular}

\section{Abstract}

There is a need for a source of particles that simulate those from nuclear reactor accidents or fallout. Studies of surface decontamination, the fate of such particles, disposal of accumulated contamination, etc., cannot be done with actual sources. Although surrogate materials exist for forensics studies, they haven't the properties needed for assessing environmental fate and decontamination efficacy. We are developing a process, using an electric arc furnace, to generate chemically, mineralogically, and physically accurate particles that are directly and reproducibly applicable to the study of fallout from various incidents. With the time and resources available, we formed melts with the arc furnace and tested several particulate sampling methods. After flowing gas over or bubbling it through the melts, particulates were produced and collected on a filter system. Either particles of the correct size or of the correct morphology were produced. Further work is required to achieve both metrics plus correct composition in a single particulate.

\begin{tabular}{|l|l|}
\hline LDRD\# 2019-0100 & Innovate \\
\hline Project Title: & FTK: The Feature Tracking Kit \\
\hline Investigators: & Hanqi Guo \\
\hline
\end{tabular}

\section{Abstract}

Scientists continually reinvent and tailor feature tracking algorithms for different applications. A generalized in situ feature tracking framework that eliminates the need for redundant efforts to develop domain specific feature tracking algorithms would be a breakthrough in data analysis and visualization. In the exascale computing era, as the gap increases between data production rates and I/O bandwidth, in situ feature tracking would make it possible to derive insights during scientific simulations and thus reduce the data to be stored. The goal of this project was to develop a generalized feature tracking kit (FTK), and we succeeded in doing so. Our FTK was demonstrated successfully for three types of data: microparticle tracking in high-speed imaging experiments; blob tracking in gyrofluid tokamak simulations; and singularity (vortex) tracking in superconductors. The novelty of the FTK is based on the generality of the framework and the ability to incorporate state-of-the-art deep learning, statistical, and topological feature tracking algorithms. Depending on application, features can be described as points, lines, surfaces, or regions. As a generalized framework, FTK incorporates a zoo of feature representations for various applications. In addition to its flexibility, FTK has high performance and will enable scalable in-situ feature tracking with the simulations that run on today's and future leadership computing facilities. 


\begin{tabular}{|l|l|}
\hline LDRD\# 2019-0104 & Innovate \\
\hline Project Title: & $\begin{array}{l}\text { Computational Framework to Characterize Extreme Events for Complex Physical } \\
\text { Phenomena }\end{array}$ \\
\hline Investigators: & $\begin{array}{l}\text { Vishwas Rao } \\
\text { Mihai Anitescu, Emil Constantinescu }\end{array}$ \\
\hline
\end{tabular}

\section{Abstract}

An ability to predict extreme events, such as cascading failures in the power grid, hurricanes, and tornadoes can have far-reaching socioeconomic impacts. State-of-the-art techniques to achieve such predictions are based on variants of Monte Carlo methods, which require large numbers of simulations to ensure accuracy; this causes extensive (often impractical) delays in prediction. We developed a new technique combining gaussian mixture models (GMM) with analytical results from stochastic processes, which enables predictions two to three orders of magnitude faster than state-of-the-art approaches without compromising accuracy. By using the GMM in conjunction with the theory of excursion probabilities (TEP) to build a Markov Chain Monte Carlo (MCMC)-based importance sampling (MCMCbased IS) algorithm to accurately compute tail probability distributions, we overcome the expense of accurately computing the tail distributions of complex physical phenomena. These computational tools can be applied to accurately estimate the risk in critical infrastructures such as the power grid. Our research has the potential to improve the long-term stability of power grids and the predictability of extreme weather events. One can also use this technique to balance risks and costs associated with the design of critical infrastructure.

\begin{tabular}{|l|l|}
\hline LDRD\# 2019-0112 & Innovate \\
\hline Project Title: & $\begin{array}{l}\text { Goal-Oriented Optimal Experimental Design Framework for Sensor Placement } \\
\text { and Acquisition of Highly-Correlated Data }\end{array}$ \\
\hline Investigators: & $\begin{array}{l}\text { Ahmed Attia } \\
\text { Emil Constantinescu }\end{array}$ \\
\hline
\end{tabular}

\section{Abstract}

Optimal design of experiments is the general formalism governing decisions about sensor placement and data collection strategies for engineered or natural experiments. The need for this formalism is prevalent in many fields, such as battery design, geosciences, and environmental and urban studies. State-of-the-art computational methods for experiment design do not accommodate correlated observations produced by many devices, such as X-ray machines, radar stations, and satellites. Discarding data correlations leads to biased results, higher expenses, and wasted resources. We developed and implemented a goal-oriented optimal experimental design framework suitable for largeto-extreme-scale problems for which measurement errors are generally correlated. The project integrates ideas from statistical modeling, machine learning, and high-performance computing to enable the design of optimal sensor placement and data collection plans that maximize the information gain at minimum computational and financial costs. Our strategy complements an ongoing project at the Atmospheric Radiation Measurement Climate Research Facility with respect to wind observations and potentially applies to the Argonne-led "Array of Things" project. Our work is also well aligned with the 
initiative to help communities tackle local challenges, improve city services, and study urban environments.

\begin{tabular}{|l|l|}
\hline LDRD\# 2019-0142 & Innovate \\
\hline Project Title: & $\begin{array}{l}\text { Statistical Multi-Scale Methods for Parameterization of Complex Physics-Based } \\
\text { Models }\end{array}$ \\
\hline Investigators: & $\begin{array}{l}\text { Julie Bessac } \\
\text { Ahmed Attia, Emil Constantinescu, Jiali Wang }\end{array}$ \\
\hline
\end{tabular}

\section{Abstract}

Numerical models are used extensively to simulate large-scale physical phenomena, such as the earth climate system (or its components), material properties, and battery designs. These models are discretized representations of true physical processes; however, a limitation is in the scales accommodated by the computational meshes used, which prevent capturing all scales of the real process. Many phenomena happen at scales finer than the ones captured by the discretization methods, and thus are not completely represented; this is called subgrid variability. Quantifying the influence of the sub-grid scales on the resolved scales is needed to better represent the entire system. Each application will have its own quirks, and there is generally no accepted subgrid variability solution across them. Coupling machine learning algorithms to robust statistical approaches allows us to attack the multiple domains of applications and potentially lead to an entire framework for subgrid variability. This project is developing a framework for bridging scales in atmospheric models by modeling the relationship between physical model characteristics at different levels. We evaluated the difference between the true fluxes driven by wind speed from a simulated climate dataset and fluxes calculated using area-averaged wind speeds. We proposed a space-time characterization of this discrepancy, conditioned on the low-resolution fields, with the view of developing a stochastic wind-flux parameterization. A locally stationary space-time Gaussian model is used to characterize and model this error process. The study was performed on high-resolution simulations on a domain that extends across the Indian Ocean into the Western Pacific.

\begin{tabular}{|l|l|}
\hline LDRD\# 2019-0153 & Innovate \\
\hline Project Title: & $\begin{array}{l}\text { Fabrication of Single Crystal and High Aspect Ratio Piezoelectric Nanofibers for } \\
\text { Nanogenerator Applications }\end{array}$ \\
\hline Investigators: & Moinuddin Ahmed \\
\hline
\end{tabular}

\section{Abstract}

We intend to grow high crystallinity and high aspect ratio piezoelectric nanofibers from solid solution $\left[\mathrm{Pb}\left(\mathrm{Mg}_{1 / 3} \mathrm{Nb}_{2 / 3}\right) \mathrm{O}_{3}\right](1-\mathrm{x})+\left[\mathrm{PbTiO}_{3}\right] \times$ (lead magnesium niobate + lead titanate or PMN-PT) to achieve a high piezoelectric coefficient. Single crystal piezoelectric material can generate higher voltage compared to polycrystalline material during mechanical deformation, and high aspect ratio nanofibers can raise the piezoelectric coefficient by factors of five to ten compared to bulk material. Piezoelectric nanofibers can be the fundamental element for an energy harvester, such as a nanogenerator, which is a building block of self-powered and autonomous devices, and implantable medical devices. PMN-PT powder was 
synthesized from a chemical reaction of an organic salt solution and thermal annealing of the solution. X-ray diffraction (XRD) of the powder confirmed a strong (110) peak, a necessity for piezoelectric nanofiber production. A polymer-based solution containing the PMN-PT powder was prepared for an electrospinner which produced polymer-based nanofibers. These were oxidized to fabricate PMN-PT nanofibers. Based on this demonstration, the efficacy of producing high aspect ratio perovskite piezoelectric nanofibers can be further explored.

\begin{tabular}{|l|l|}
\hline LDRD\# 2019-0246 & Innovate \\
\hline Project Title: & $\begin{array}{l}\text { Developing Capability to Generate High Resolution Agronomic and Economic } \\
\text { Decision Variables for Creating Win-Win for Bioenergy Crops and Water Quality }\end{array}$ \\
\hline Investigators: & $\begin{array}{l}\text { Shruti Khadka Mishra } \\
\text { Umakant Mishra, Jules Cacho }\end{array}$ \\
\hline
\end{tabular}

\section{Abstract}

Balancing the food, energy, water nexus in the agricultural land of the U.S. Midwest has been a challenge for scientists and decision makers. While the programs implemented focus on increasing efficiency of nutrient use and incentivizing reduction of nutrient loss, a lack of critical bio-physical, economic, and institutional information constrain site-specific programs. We built a capability to generate high resolution (30-meter) biophysical and economic data pertaining to nutrient reduction options including through the introduction of bioenergy crops. We completed data collection, cleaning and processing, including soil properties, weather data, digital elevation model data, and land use land cover data. We also obtained data on grain and bioenergy crops production, crop area, costs, and price. With those data, we generated a high-resolution map for marginal land in Illinois including for the corngrowing area. We developed an economic analysis model and, lastly, we identified the agents and developed a framework for agent-based modeling.

\begin{tabular}{|l|l|}
\hline LDRD\# 2019-0292 & Innovate \\
\hline Project Title: & Computational Design of Li-ion Cathodes Based on Transition Metal Oxysulfide \\
\hline Investigators: & $\begin{array}{l}\text { Liang Li } \\
\text { Maria Chan }\end{array}$ \\
\hline
\end{tabular}

\section{Abstract}

The purpose of this project is to perform ab-initio computational investigations, in combination with spectroscopy simulations, to search for and design transition metal (TM) oxysulfide systems that can be used as high-capacity lithium-ion batteries (LIB) cathode materials. The technological advancement of cathode materials is the main driver for next-generation high-performance, low-cost, and environmentally benign lithium-ion batteries. However, the heavy reliance on transition metal oxides in today's commercial lithium-ion batteries poses several fundamental challenges. The TM oxysulfides, on the other hand, have low-cost, high charge/discharge rates, and potentially high theoretical capacity as a result of multiple cationic and anionic redox centers. In addition, the TM oxysulfides may help to overcome the poor cyclability in TM-sulfide LSBs (lithium-sulfur batteries) caused by the large volume 
change during Li insertion/removal. We are performing high-throughput screening of 3d-TM oxysulfides using materials databases. We screen for composition, band gap, formation energy, and thermodynamic stability. Our first-principles calculations of structural, energetic and electronic properties are followed by prediction of electrochemical and kinetic properties. Our computer-aided characterization results in prediction of spectroscopic features to be found in X-ray characterization of the materials. These results are expected to stimulate and guide benchtop synthesis and characterizations of this new type of electrode material.

\begin{tabular}{|l|l|}
\hline LDRD\# 2019-0316 & Innovate \\
\hline Project Title: & Magnetically-Enforced Microbial Scavengers \\
\hline Investigators: & $\begin{array}{l}\text { Gasper Kokot } \\
\text { Martyna Michalska }\end{array}$ \\
\hline
\end{tabular}

\section{Abstract}

Biomanufacturing's contribution to product development is growing-leading to the need to improve downstream processing, which represents 85 percent of the production cost. Intracellularly produced materials often require breaking cells open. Although a high-pressure homogenizer is typically used, it has drawbacks (e.g., heating, a need for multiple cycles, and extreme shear forces) that limit its use on fragile biomolecules. For such sensitive biomolecules such as deoxyribonucleic acid (DNA), some proteins, and biofuels, chemical methods are often applied. They raise costs and increase waste generation. We are developing an efficient and easily scalable method for mechanical cell-lysis (disruption) based on use of magnetically responsive "nanorough" microparticles of iron-cobalt (FeCo) alloy. These are flower-like microparticles with their surface nanotopology characterized by extremely sharp edges. Similar to the bead grinding technique, our method vigorously mixes the medium with the suspended bacterial cells and our designed solid particles. The stirring mechanism relies on the particles' magnetic properties and is driven by a triaxial magnetic field. Triaxial magnetic mixing is scalable, tunable and high speed at modest magnetic field strength $(<\sim 10 \mathrm{mT})$. The microparticle roughness is expected to mechanically damage cell membranes. Our method should result in (1) complete lysis within seconds (compared to minutes with current methods), (2) the ability to operate in a semicontinuous flow, and (3) a means by which to process denser cell suspensions. Thus, the overall cost of cell lysis in a bioreactor is reduced. 


\begin{tabular}{|l|l|}
\hline LDRD\# 2019-0392 & Swift \\
\hline Project Title: & $\begin{array}{l}\text { Enhancing Simulation Tools for Hypersonic Flight Through Improved Fuel } \\
\text { Injection Modeling in Air-Breathing Jet Engines }\end{array}$ \\
\hline Investigators: & $\begin{array}{l}\text { Gina Maureen Magnotti } \\
\text { Sibendu Som }\end{array}$ \\
\hline
\end{tabular}

\section{Abstract}

To address the challenges of hypersonic flight, a predictive computational design tool is needed that can advance development of propulsion systems. Fuel injection system design is critical, because, under extreme supersonic crossflow conditions, it forms the combustible fuel-air spray mixture needed for efficient and clean combustion in air-breathing propulsion devices. Key to predicting fuel injection is a computational fluid dynamics framework that can incorporate the details of an injector geometry and its influence on internal flow and external spray. Based on our expertise in fuel injection modeling for automotive applications, we extended the predictive capability of our simulation tools to address injection conditions relevant to commercial aviation and defense applications. We have observed from our modeling experience that surface roughness and manufacturing tolerances have a strong influence on resultant fuel spray. We had developed the capability of using x-ray measurements from the Advanced Photon Source to iA key uncertainty in developing earth system models is from deep convection, which is vital for accurately representing the atmospheric water cycle. Current $12 \mathrm{~km}$ resolution simulations are too coarse for risk assessment, especially at neighborhood scale, and have large biases due to the use of convective parameterization. We are producing a high-fidelity regionalscale climate model at a spatial resolution of $4 \mathrm{~km}$ that fully resolves the convective process (referred to as convective-permitting) and thus improves prediction of hydrological events. This project builds on the database of model output developed over the past few years and establishes a progression of datasets showing an improvement over our current data products. We tested convective-permitting modeling by focusing on the entire North America region. This is the largest domain being tested in the research community with such a high spatial resolution. With increased availability of the high-performance computing (HPC) resource at Argonne, developing this high-resolution model dataset has become tractable. The output can be used in many other research areas, such as hydrology, ecology, agriculture, and infrastructure.nform the modeled computational domain. A simulation approach was developed and validated to understand the internal flow development and its coupling with the external spray. Recent application of X-ray diagnostics to jets in subsonic crossflow included characterization of the jet structure, with particular attention paid to the liquid mass distribution in the near-nozzle region.

Comparisons between the predicted and experimentally measured liquid mass distributions were used to validate the modeling approach. The influence of surface finish of the internal surface of the injector on the spray characteristics was then explored computationally. Surface roughness was synthetically imposed in various regions of the injector and the exit condition of the injector nozzle was analyzed. Although the mean jet trajectory was found to be largely insensitive to the presence of surface roughness in the injector, the unsteadiness of the jet is affected. 


\begin{tabular}{|l|l|}
\hline LDRD\# 2019-0395 & Swift \\
\hline Project Title: & $\begin{array}{l}\text { Generation of a Next Level Dataset for Regional Scale Climate Modeling: } \\
\text { Convective Resolving Spatial Scales }\end{array}$ \\
\hline Investigators: & $\begin{array}{l}\text { Jiali Wang } \\
\text { Rao Kotamarthi }\end{array}$ \\
\hline
\end{tabular}

\section{Abstract}

A key uncertainty in developing earth system models is from deep convection, which is vital for accurately representing the atmospheric water cycle. Current $12-\mathrm{km}$ resolution simulations are too coarse for risk assessment, especially at neighborhood scale, and have large biases due to the use of convective parameterization. We are producing a high-fidelity regional-scale climate model at a spatial resolution of $4 \mathrm{~km}$ that fully resolves the convective process (referred to as convective-permitting) and thus improves prediction of hydrological events. This project builds on the database of model output developed over the past few years and establishes a progression of datasets showing an improvement over our current data products. We tested convective-permitting modeling by focusing on the entire North America region. This is the largest domain being tested in the research community with such a high spatial resolution. With increased availability of the high-performance computing (HPC) resource at Argonne, developing this high-resolution model dataset has become tractable. The output can be used in many other research areas, such as hydrology, ecology, agriculture, and infrastructure.

\begin{tabular}{|l|l|}
\hline LDRD\# 2019-0397 & Swift \\
\hline Project Title: & $\begin{array}{l}\text { Combining High-Performance Computing, Artificial Intelligence, and Agent- } \\
\text { Based Models to Combat Misinformation Campaigns }\end{array}$ \\
\hline Investigators: & Matthew Wolf \\
\hline
\end{tabular}

\section{Abstract}

Social media is being used to spread inaccurate and incomplete information. This project intends to use artificial intelligence to identify key factors associated with influence campaigns and to calculate a credibility score for each piece of information. Agent-based models (ABM) are used to capture the dynamics of information diffusion, behavior change, and opinion dynamics in response to misinformation. We developed a mathematical basis for an ABM of computational propaganda to study the spread of disinformation among a population. Our initial prototype focused on anti-vaccination disinformation using open-source software as a proof-of-concept. More in-depth analysis using realworld social media data is ongoing. What sets our approach apart from other efforts is that we apply our methodology across multiple social media platforms (e.g., Twitter, Facebook, Reddit, print media) as opposed to a single platform. Other misinformation research focuses solely on detection; our approach includes both a detection and modeling solution. Our methodology will help everyone from individual users to public and private institutions recognize, understand, expose, and counter misinformation campaigns. 


\begin{tabular}{|l|l|}
\hline LDRD\# 2019-0398 & Swift \\
\hline Project Title: & Laser Joining of Dissimilar Metals \\
\hline Investigators: & $\begin{array}{l}\text { Xuan Zhang } \\
\text { Wei-Ying Chen }\end{array}$ \\
\hline
\end{tabular}

\section{Abstract}

We explored the joining process of dissimilar metals by laser additive manufacturing (AM). The targeted application is the cladding components in nuclear reactors, specifically, molten salt reactors in which the primary coolant salt tends to leach chromium from a reactor's exposed structural materials. Compared to current cladding techniques, laser AM has the advantage of being flexible with geometry and composition, as well as providing better interface adhesion due to melting of materials at the interface with a smaller heat input that results in less microstructural impact. We planned to study two materials systems: 316 stainless steel (base) + nickel-based super alloy (cladding) and Alloy 617 (base) + tungsten (cladding). The goal was to identify the optimal manufacturing conditions to achieve satisfactory interface properties. We have preliminary work on a 316 stainless steel + nickel super alloy system showing that such a joining via laser AM is possible, and that the laser parameters critically affect the joining process and defect formation. We assessed results of the process through the advanced imaging and diffraction techniques available at the Advanced Photon Source (APS). We utilized the ultrafast x-ray imaging at the APS for in-situ observation of the laser AM process in an effort to develop a fast materials development paradigm. Following the APS work, samples were studied by electron microscopy for microstructural characterization and then mechanical testing for property screening. The results showed that the laser AM process successfully joined Inconel 718 with 316 stainless steel with a strong interface bond. The elemental distribution around the interface was inhomogeneous, resembling the liquid metal flow during the joining process. The ultrafast x-ray imaging helped to identify the optimum process window towards defect-free products.

\begin{tabular}{|l|l|}
\hline LDRD\# 2019-0404 & Swift \\
\hline Project Title: & $\begin{array}{l}\text { Design of a Modular Ocean Mining System for Seawater Rare Element } \\
\text { Extraction and Desalinization }\end{array}$ \\
\hline Investigators: & $\begin{array}{l}\text { Mark Christian } \\
\text { Meltem Urgun Demirtas, Santanu Chaudhuri }\end{array}$ \\
\hline
\end{tabular}

\section{Abstract}

We developed preliminary system designs and economic analysis related to the Modular Ocean Mining System (MOMS), a scalable and modular marine hydrokinetic system capable of desalinizing seawater and extracting specified elements from the concentrated waste brine produced by the process. This research couples desalinization and marine element extraction: two concepts that have been pursued to varying degrees separately, but have not been found economically viable. By coupling the two systems, the benefits of both are achievable while the disadvantages of each can be largely mitigated. By using a combination of nano filters and reverse osmosis filters (reducing necessary energy) and using ocean motion to provide the energy and pressure required, the technology becomes more cost competitive. The technique avoids pitfalls in marine element extraction by extracting the element(s) directly from the concentrated brine and using marine and hydrokinetic-derived energy to power the process. We 
demonstrated how the coupled MOMS has the economic and technical viability to provide broad system wide benefits (ow cost - high pressure; fresh water availability; and alternative element sourcing). Overall, this effort finds a positive revenue to cost ratio of the system with dependency on appropriate wave frequency and economies of scale for desalinization and capital costs.

\begin{tabular}{|l|l|}
\hline LDRD\# 2019-0408 & Swift \\
\hline Project Title: & $\begin{array}{l}\text { Advanced Moderation Module (AMM) Development for Micro-Very-High- } \\
\text { Temperature Reactors }\end{array}$ \\
\hline Investigators: & $\begin{array}{l}\text { Yinbin Miao } \\
\text { Nicolas Stauff, Sumit Bhattacharya }\end{array}$ \\
\hline
\end{tabular}

\section{Abstract}

The U.S. nuclear industry is focusing on micro-reactors, which require cutting-edge technologies to realize compactness, efficiency and safety. Micro gas-cooled-reactors based on very-high-temperature reactor technology (beyond $900^{\circ} \mathrm{C}$ ) would significantly benefit from moderation modules that are more efficient than conventional graphite because they would enable both improved core compactness and performance. The objective of our project is development of a high-performance compact moderation module. The advanced moderation module (AMM) we envision here uses yttrium hydride encapsulated by a niobium liner, atomic-layer-deposition of an alumina $\left(\mathrm{Al}_{2} \mathrm{O}_{3}\right)$ surface coating, and a silicon carbide/silicon carbide cladding for maintaining the hydrogen-to-metal ratio at high operating temperatures. The AMM would be inserted into the graphite matrix to boost neutron moderation. We performed neutronics and thermomechanical simulations to demonstrate the advantages of using the AMM in micro-reactors and to help guide material selection. The key part of the AMM, the liner with a hydrogen permeation coating, was developed and tested using a hydrogen permeation measurement device that was established as part of this project.

\begin{tabular}{|l|l|}
\hline LDRD\# 2019-0409 & Swift \\
\hline Project Title: & Open Source Atmospheric Data Community Toolkit \\
\hline Investigators: & $\begin{array}{l}\text { Adam Theisen } \\
\text { Scott Collis, Nicki Hickmon, Zachary Sherman, Robert Jackson }\end{array}$ \\
\hline
\end{tabular}

\section{Abstract}

The Atmospheric data Community Toolkit (ACT) was developed as an open-source Python repository to help scientists easily work with atmospheric time series datasets and share their code with the community. It has been licensed by Argonne and is online at https://github.com/ANL-DIGR/ACT. It was adopted for use by the Atmospheric Radiation Measurement User Facility (ARM) Data Quality Office. As an open-source online resource, the toolkit is receiving input from its community of users. Researchers tend to write their own code to perform basic functions for reading, visualizing, and analyzing data leading to redundant efforts by the research community. Open-source software, such as the toolkit produced here, bypasses that process and moves the effort toward capabilities that can be used by that broader scientific community which in turn, can contribute to and collaborate on efforts to improve the 
process. Our toolkit has a solid framework that will be easy to use and provide clear benefit worthy of the investment in participating, a clear requirement for any software to be adopted by a community. There has also been growing interest in the broader science community for this effort and we expect that it will continue to expand.

\begin{tabular}{|l|l|}
\hline LDRD\# 2019-0410 & Swift \\
\hline Project Title: & A Novel Security Analysis Toolbox for National Grid Resilience Modeling \\
\hline Investigators: & $\begin{array}{l}\text { Feng Qiu } \\
\text { Bai Cui, Rui Yao }\end{array}$ \\
\hline
\end{tabular}

\section{Abstract}

Power system operators rely on two types of analysis, steady-state and dynamic security assessment (S/DSA), for assessing resilience (a system's capacity to resist, respond to, and recover from disruption by extreme events). Existing tools handle routine security analysis well under normal operating conditions. Under stressed scenarios, however, studies of system security and reliability (i.e., resilience analysis) using existing tools experience convergence and numerical issues. These significantly impact an operator's assessment of system resilience. We have developed novel methodologies and algorithms (i.e., semi-analytical simulation and convex relaxation optimal power flow) to address these numerical issues. Semi-analytical simulation provides arbitrarily high-order approximate solutions efficiently, leading to robust numerical stability and strong computational efficiency. Advances in convex relaxation optimal power flow provide tractable computational methods for global optimal power flow solutions, as well as opening the door to numerically stable methods. Our results suggest that the two approaches have great potential to address the numerical issues in power system resilience analysis. We also worked on a modeling framework and computational architecture for dissemination of our methods to industry and the R\&D community.

\begin{tabular}{|l|l|}
\hline LDRD\# 2019-0412 & Swift \\
\hline Project Title: & State Preservation for Deep Learning Applications \\
\hline Investigators: & Bogdan Nicolae \\
\hline
\end{tabular}

\section{Abstract}

Deep learning applications are rapidly gaining adoption both in industry and the scientific computing. The ability to quickly save and restore the internal state of a learning model is critical to enabling fault tolerance and to facilitating possible later reuse for training strategies for such applications. However, creating this ability is not trivial, given the many models such applications need to explore and the limited input/output (I/O) bandwidth of supercomputers. We explored design and development of efficient state preservation techniques in the context of large-scale deep learning applications. The ability to leverage idle $\mathrm{I} / \mathrm{O}$ and network resources during feed-forward; the flexibility to schedule backpropagation and/or couple it with additional operations that modify tensors; and the opportunity to perform additional immutable asynchronous operations in the background on the tensors during the training with minimal interference, are all key findings from our study on how best to achieve state 
preservation in deep learning model configurations. In addition, our findings were used to develop an advanced checkpointing strategy for VELOC (Very Low Overhead Checkpointing System), a checkpointing system supported by the Exascale Computing Program (ECP). Representative use cases of deep learning obtained from the CANDLE (Collaborative and Network Distributed Learning Environment) team were used to evaluate the benefits of our approach.

\begin{tabular}{|l|l|}
\hline LDRD\# 2019-0437 & Swift \\
\hline Project Title: & An Internet Islanding Approach to Deterrence of Nation-State Adversaries \\
\hline Investigators: & $\begin{array}{l}\text { Nate Evans } \\
\text { Benjamin Blakely }\end{array}$ \\
\hline
\end{tabular}

\section{Abstract}

Ascertaining the credibility and seriousness of a threat made by the United States to cut itself off from the global Internet in case of attack - cyber or otherwise - is a complex multifaceted task. Russia has recently introduced legislation that would grant its government the power to cut the country off from the global Internet. In this project, we evaluate the factors that would influence such a step in the United States. Our research drew upon open source information regarding trans-national U.S. internet connectivity, facilities with high concentrations of internet service providers or web applications, legal issues regarding government intervention in privately-owned infrastructure, and the economic impact to both wholly domestic and multi-national corporations of such a disconnection. As a practical matter, many tens of submarine fiber optic cables as well as terrestrial and aerial/satellite links connect the US network internationally. These would need to be partially or fully severed. A nationwide program implemented federally against a largely privately owned enterprise would confront the Posse Comitatus Act, if the US military were employed. Uniform state-by-state compliance would be problematic. The economic impact on business and the national GDP would be substantial, both due to loss of domestic connectivity and loss of international trade. Our goal has been to determine the feasibility and potential impact of such a disconnection and to use established concepts of deterrence theory to consider whether the threat to disconnect is likely to have meaningful impact on adversarial behavior. A proprietary White Paper with our results has been distributed to our university and government partners.

\begin{tabular}{|l|l|}
\hline LDRD\# 2019-0481 & Swift \\
\hline Project Title: & $\begin{array}{l}\text { Cathode Composite Electrode Composed of 1 Micron-size Single Crystal LLZO } \\
\text { Filler and Core(NMC811)-Shelled(LLZ) Cathode }\end{array}$ \\
\hline Investigators: & YoungHo Shin \\
\hline
\end{tabular}

\section{Abstract}

Lithium lanthanum zirconium oxide (LLZO), with nominal composition $\mathrm{Li}_{7} \mathrm{La}_{3} \mathrm{Zr}_{2} \mathrm{O}_{12}$, is a promising solid electrolyte for Li-based batteries due to its high Li-ion conductivity and chemical stability with respect to lithium. We address challenges behind the manufacturing of one-micron-size single crystal LLZO solid electrolyte used for improving the interfacial coherence by filling in the voids among the particles in a 
cathode composite electrode. We also explored production of solid-solid bilayer cathode particles using supercritical hydrothermal synthesis. This work includes the synthesis of a core (NMC811)-shell (LLZO) cathode, which is a solid-solid bilayer structured particle with low porosity, high interfacial coherence, and good mechanical and chemical stability, for advanced solid-state batteries. NMC811 is a nickel-rich layered oxide of composition $\mathrm{LiNi}_{0.8} \mathrm{Mn}_{0.1} \mathrm{Co}_{0.1} \mathrm{O}_{2}$. One-micron-level single-crystal-like pure LLZO and 0.2 Al-doped LLZO, with uniform Al distribution, were successfully produced through synthetic experiments using various starting chemicals and reaction processes. Through various reaction additives, control of particle size and morphology of LLZO was identified. Reproducibility of material synthesis and accurate stoichiometry of the products was realized through optimization of the reaction process. In the course of these studies, a hydro/solvothermal synthesis platform was developed to synthesize solid oxide electrolytes of various shapes, sizes, and compositions.

\begin{tabular}{|l|l|}
\hline LDRD\# 2019-0482 & Swift \\
\hline Project Title: & $\begin{array}{l}\text { Development of Dense LLZO Solid-State Electrolytes from Ultrafine-Grain } \\
\text { Nanofibers }\end{array}$ \\
\hline Investigators: & $\begin{array}{l}\text { Yuepeng Zhang } \\
\text { Peter Zapol, Sanja Tepavcevic, Yisi Zhu, Erik Dahl }\end{array}$ \\
\hline
\end{tabular}

\section{Abstract}

Our project addressed the challenge of poor processability associated with brittle ceramic solid electrolytes prepared by high-temperature sintering processes. We developed a low temperature synthesis path that can fabricate highly conductive nanofiber-based solid electrolytes to advance solidstate lithium battery R\&D and manufacturing. Our key accomplishment is a methodology that produces by electrospinning, high ionic conductivity cubic phase $\mathrm{Li}_{7} \mathrm{La}_{3} \mathrm{Zr}_{2} \mathrm{O}_{12}$ (LLZO) nanofibers at a temperature that is $400-500^{\circ} \mathrm{C}$ lower than that used by the state-of-art powder sintering process. This achievement can significantly reduce thermal budget and manufacturing cost. By developing ultrafine-grain nanofiber LLZO, we gain flexibility in the densification process, significantly improving solid electrolyte density and conductivity. The nanofiber electrolytes show great potential in fast charging due to their long, continuous lithium-ion transport path. Our method is cost-effective, energy-efficient, and scalable (because of roll-to-roll nanofiber synthesis process based on electrospinning technique).

\begin{tabular}{|l|l|}
\hline LDRD\# 2019-0483 & Swift \\
\hline Project Title: & Aerosol Deposition of Dense Ceramic Layers for All-Solid-State Batteries \\
\hline Investigators: & $\begin{array}{l}\text { Eungje Lee } \\
\text { Tae Lee }\end{array}$ \\
\hline
\end{tabular}

\section{Abstract}

All-solid-state batteries based on ceramic lithium conductors have attracted much interest as safe and high-energy alternatives to the current lithium-ion batteries, because ceramic electrolytes are nonflammable and have high ionic conductivity. Despite significant progress in the materials development of the ceramic solid electrolytes and electrodes, the performance of all-solid-state 
batteries is still far inferior to that of conventional lithium-ion batteries mainly because of issues in ceramic processing. Conventional ceramic techniques require complex high-temperature and highpressure processes, which are not scalable or cost-effective. Aerosol deposition (AD) is a recently developed method that forms dense ceramic films with high film-fabrication speed (greater than five micrometers/minute). We are developing an $A D$ thin-film fabrication process to overcome the issues in traditional ceramic processing. The $A D$ method is a scalable technology that enables impact consolidation (mechanical sintering) of ceramic particles into a dense membrane without a heating process (i.e., thermal sintering). We are testing the feasibility of the room-temperature AD method in fabricating a dense, micron-thick solid-state electrolyte membrane. We fabricated a fully dense, 10-20 micrometer-thick cubic $\mathrm{Li}_{7} \mathrm{La}_{3} \mathrm{Zr}_{2} \mathrm{O}_{12}$ (c-LLZO) solid-electrolyte membrane at room temperature. The effective film conductivity was about 10-5 siemens/cm which is comparable to typical c-LLZO pellets of millimeter thickness fabricated by the high-temperature sintering process. When applied to a solidstate battery, this micron-thick c-LLZO membrane will reduce the Li-diffusion length and the overall cell resistance significantly.

\begin{tabular}{|l|l|}
\hline LDRD\# 2019-0486 & Swift \\
\hline Project Title: & $\begin{array}{l}\text { Rapid Microwave-Assisted Sintering of LLZO Solid State Electrolyte: An Energy- } \\
\text { Efficient Manufacturing Alternative }\end{array}$ \\
\hline Investigators: & $\begin{array}{l}\text { Jessica Durham } \\
\text { Albert Lipson }\end{array}$ \\
\hline
\end{tabular}

\begin{abstract}
Processing is one of the main issues hindering commercialization of solid-state electrolytes. To prevent lithium dendrite propagation and facilitate high ionic conductivity, solid-state electrolyte sheet densities near theoretical are required but cannot be achieved using conventional sintering techniques. These highly dense sheets act as separator and ion conductor between electrodes. Conventional sintering techniques for ceramic electrolytes are also low throughput and with long sintering times (greater than 10 hours) at high temperature (greater than $1,000^{\circ} \mathrm{C}$ ). Lithium loss from the structure also occurs under these conditions. Our project introduces an alternative: microwave sintering. Microwave sintering is attractive because it provides ultra-high densification in less than $\mathbf{3 0}$ minutes, consumes less energy, is applicable to large samples, and can produce ceramic electrolytes with improved conductivity and mechanical properties. Solid state electrolyte pellets composed of LLZO (lithium lanthanum zirconium oxide) demonstrated up to a $20 \%$ increase in density when sintered in a microwave furnace versus a conventional muffle furnace under the same conditions. In addition, microwave sintering increased conductivity of LLZO pellets by nearly an order of magnitude for samples calcined at $1050^{\circ} \mathrm{C}$. These results open an opportunity to achieve higher density and improved ionic conductivity in LLZO and other solid-state electrolyte candidates.
\end{abstract}




\begin{tabular}{|l|l|}
\hline LDRD\# 2019-0487 & Swift \\
\hline Project Title: & $\begin{array}{l}\text { Development of Conductive Solid-State LLZO Electrolyte by Hot-pressing of } \\
\text { Electrospun Precursor LLZO Nanofibers }\end{array}$ \\
\hline Investigators: & $\begin{array}{l}\text { Dileep Singh } \\
\text { Erik Dahl, Yuepeng Zhang }\end{array}$ \\
\hline
\end{tabular}

\section{Abstract}

The focus of our work is synthesis of a dense $\mathrm{Li}_{7} \mathrm{La}_{3} \mathrm{Zr}_{2} \mathrm{O}_{12}$ (LLZO) electrolyte using amorphous electrospun nanofibers. This scoping study demonstrated the densification of LLZO nanofibers while producing a cubic phase with high ionic conductivity. We demonstrated densification of LLZO precursor powder material into a dense ceramic. Two techniques, hot pressing and field-assisted sintering technology were used. Both techniques resulted in a final product with densities $>98 \%$ of theoretical. Field assisted sintering technology has advantages such as significantly less time to process leading to minimal loss of lithium from the ceramic. This demonstration points to development of better engineered microstructures for solid-state battery materials with improved electrical and mechanical performance.

\begin{tabular}{|l|l|}
\hline LDRD\# 2019-0494 & Swift \\
\hline Project Title: & Manufacturing Processes for Lithium Metal Using Advanced Electrodeposition \\
\hline Investigators: & $\begin{array}{l}\text { Edward Barry } \\
\text { Donghyeon Kang }\end{array}$ \\
\hline
\end{tabular}

\footnotetext{
Abstract

Industrial processes for production of lithium-metal thin films (under 20 micrometer thickness) for solidstate batteries (SSBs) suffer from low productivity and high-energy input, inevitably resulting in high production costs. A facile and inexpensive production alternative is needed. We have proposed an innovative manufacturing process based on advanced electrodeposition that can simultaneously produce protective, stable solid electrolyte interphase coatings and battery-grade lithium-metal thin films at production scale. We demonstrated control over lithium film morphology and thickness, under certain sets of conditions and controlled electrodeposition parameters (e.g., voltage and current density) as well as solution conditions (e.g., additives). Quantitative comparison of the lithium metal thin films developed here with those of a commercial supplier demonstrated a marked increase in cycling capacity in coin cell testing.
} 


\begin{tabular}{|l|l|}
\hline LDRD\# 2019-0498 & Swift \\
\hline Project Title: & $\begin{array}{l}\text { Fabrication of Large Area Dense Separator via Tape Casting and Pressure-less } \\
\text { Sintering }\end{array}$ \\
\hline Investigators: & $\begin{array}{l}\text { Xiaoping Wang } \\
\text { John Carter }\end{array}$ \\
\hline
\end{tabular}

\section{Abstract}

Next generation all solid-state Li-ion batteries require a scalable manufacturing process for large area dense separators and thus large format batteries. We used doped LLZO $\left(\mathrm{Li}_{7} \mathrm{La}_{3} \mathrm{Zr}_{2} \mathrm{O}_{12}\right)$ combined with the well-established industrial technique, tape casting, to generate free-standing, thin, dense electrolyte separators that are less than 50 micrometers thick, are one-quarter the size of an automotive pouch cell, and should have Li-ion conductivity on the order of 10-3 siemens/cm. This was investigated using nano-size powders generated by flame spray pyrolysis at Argonne's Materials Engineering Research Facility (MERF), attending to chemistry (binder, plasticizer, dispersant, and solvent), rheology, solid loading and viscosity of the electrolyte powder slurry for better control of the densification/sintering process conditions. Five MERF nano-sized powders with varied sizes and chemical compositions (consisting mainly of $\mathrm{La}_{2} \mathrm{Zr}_{2} \mathrm{O}_{7}$ and $\mathrm{Li}_{2} \mathrm{CO}_{3}$ ) were used to formulate slurries with desired properties. This resulted in green tapes made by the tape casting process displaying uniform thickness and uniformity of solid powder distribution, plus good peeling ability from preselected substrates. Thermal gravimetric studies of the green tapes determined the chemical and thermal events which were utilized to the guide sintering. Various heating profiles, atmospheres, and types and weights of setters were evaluated for the sintering. Sintered plates of different sizes and shapes and resealable flatness were realized. Ultimate density and ionic conductivity figures of merit await further development.

\begin{tabular}{|l|l|}
\hline LDRD\# 2019-0501 & Swift \\
\hline Project Title: & $\begin{array}{l}\text { Generating Training Datasets for Developing a Deep Learning-Computer Vision } \\
\text { Framework to Monitor Avian Interactions with Solar Energy Facility } \\
\text { Infrastructure }\end{array}$ \\
\hline Investigators: & $\begin{array}{l}\text { Leroy Walston } \\
\text { Adam Szymanski, Yuki Hamada }\end{array}$ \\
\hline
\end{tabular}

\section{Abstract}

Effective mitigation of avian mortality at utility-scale solar facilities is hindered by incomplete understanding of the phenomenon because of a lack of sufficient and timely data on the frequency and effects of avian-solar interactions. Autonomous avian detection is needed to fully understand the phenomenon, because the cost of data collection in the field is high. The capability of deep learning and computer vision approaches to autonomously detect and classify flying objects using upward-looking cameras has already been demonstrated. Obtaining a large volume of training data requires a sizable investment in time and resources. We pursued obtaining video data of bird interactions with solar photovoltaic panels as a preliminary training dataset. In summer 2019 we collected video training data of avian-solar interactions at the operational 30-kW PV facility located in Argonne's Smart Energy Plaza. These data should lead to more extensive work using a machine learning $(\mathrm{ML})$ approach with a distributed camera network (incorporating oblique- and horizontal-viewing true-color and thermal 
infrared cameras to autonomously collect and analyze images) and edge computing to detect birds, and classify their activities at solar facilities under day- and night-time conditions.

\begin{tabular}{|l|l|}
\hline LDRD\# 2019-0506 & Swift \\
\hline Project Title: & CFD modeling of Rotating Detonation Engines (RDE) \\
\hline Investigators: & $\begin{array}{l}\text { Sibendu Som } \\
\text { Pinaki Pal }\end{array}$ \\
\hline
\end{tabular}

\section{Abstract}

Detonation based propulsion has received a great deal of attention as a potential means to achieve significant improvement in the performance of air-breathing and rocket engines. Detonative combustion mode is particularly interesting due to the resulting pressure gain from reactants to products, faster heat release, decreased entropy generation, more available work and higher thrust compared to conventional deflagrative combustion. Rotating detonation engine (RDE) is one such novel combustor concept. RDE performance is governed by a large number of design parameters and operating conditions. RDEs are challenging to study experimentally due to the harsh environment in the combustor chamber comprising supersonic flows, high-frequency oscillations, high pressures and temperatures. Multidimensional modeling and computational fluid dynamics (CFD) can complement engine experiments and be a tool for more insight into RDE combustion dynamics, enabling optimization of critical design parameters that influence RDE operation and performance. We developed new large eddy simulation- (LES-) based modeling capabilities to simulate rotating detonation engines fueled by both hydrogen and ethylene. A diagnostic tool known as chemical explosive mode analysis (CEMA) was applied to the simulation data to investigate RDE combustion behavior. In addition, a sparse stiff chemistry solver was coupled with CFD to accelerate chemistry calculations in the RDE simulations. This work demonstrated novel modeling capabilities for simulating advanced combustion devices in aerospace and defense applications.

\begin{tabular}{|l|l|}
\hline LDRD\# 2019-0507 & Swift \\
\hline Project Title: & Data Enabled Catalyst Discovery \\
\hline Investigators: & Rajeev Surendran Assary \\
\hline
\end{tabular}

\section{Abstract}

Our research focuses on homogenous organometallic catalysts that provide a large exploration space of ligands and metals for developing processes for selective bond cleavage as well as the complex reaction networks resulting from them. The goal of this project is to design and discover efficient catalysts for carbon-carbon bond cleavage reactions relevant to polystyrene upcycling reactions. We established a robotic setup for the specific chemical transformation and evaluated and eliminated one catalyst, due to $\mathrm{CO}_{2}$ environmental concerns. Our experiments also identified a catalyst-ligand combination for density functional theory (DFT) simulations. To enable the experimental efforts, we have simulated the free energy profile for direct oxidative addition (bromo-phenol to biphenyl) and performed about 200 additional DFT simulations. We determined that the direct oxidative addition of substrate to the catalyst 
is a rate controlling step. Additional simulations using several metals and ligands to create a computational library for identification of optimum catalysts is a desirable next step.

\begin{tabular}{|l|l|}
\hline LDRD\# 2019-0508 & Swift \\
\hline Project Title: & Al-Coupled Experiment and Simulation to Create Tailored 3D Auxetic Materials \\
\hline Investigators: & $\begin{array}{l}\text { Benjamin Blaisik } \\
\text { Logan Ward, Marcus Schwarting, lan Foster, Juan De Pablo, Nicholas Jackson }\end{array}$ \\
\hline
\end{tabular}

\section{Abstract}

Our goal was to prepare to build a prototype automated discovery capability driven by artificial intelligence (Al) for materials, here defined as a capability that couples machine learning models, simulation, automated material assembly, and automated experimental material characterization in a feedback loop. This allows learning the details of combining simulation and experiment with active learning $(\mathrm{AL})$ for selection of subsequent experiments and can result in a new experimental setup capable of being driven by the AL model. The envisioned experimental setup consists of a 3D material printing station, capable of printing soft materials, coupled with a programmable robotic arm and characterization equipment to enable automated testing of the materials. Our initial target is discovery and testing of 3D auxetic metamaterials. We obtained and commissioned a set of general robotics equipment including six robotic arms, three conveyor belts, two soft material 3D printers, and more. These are now ready for prototype setup to autonomously explore metamaterials and also to investigate the data infrastructure needed to speed deployment of automated labs in the future.

\begin{tabular}{|l|l|}
\hline LDRD\# 2019-0509 & Swift \\
\hline Project Title: & Al Robotic Platform for Solution-Processable Materials \\
\hline Investigators: & $\begin{array}{l}\text { Jie Xu } \\
\text { Young Soo Park, Logan Ward, Angel Yanguas-Gil, Pierre Darancet, } \\
\text { Subramanian Sankaranarayanan }\end{array}$ \\
\hline
\end{tabular}

\footnotetext{
Abstract

We have designed and developed an automated high-throughput solution-processing and characterization platform. It can automatically prepare polymer solutions, cast them into films, and perform annealing treatment as well as other processing steps. It can characterize the films' morphological properties (using ultra-violet (UV)-spectroscopy), evaluate their electrical properties and log the resulting data. Achieving target properties in materials through solution-processing is challenging due to the extremely large processing parameter space, the highly non-linear growth processes, and the strong correlations between a material's morphology and its function. Our progress has addressed this challenge and prepared a path forward for integration of a machine learning method with this automated platform to simultaneously analyze the result and determine what processing condition is for the next loop. The combination of automated robotic machinery, physics-guided artificial intelligence (Al) feedback, and high throughput synthesis will accelerate the pace of solution-processable materials discovery. Furthermore, additional characterization approaches (e.g. electrical characterization, $x$-ray
} 
characterization) and synthetic data from simulation methods can be incorporated to enhance the effectiveness of the entirely automated processes.

\begin{tabular}{|l|l|}
\hline LDRD\# 2019-0510 & Swift \\
\hline Project Title: & $\begin{array}{l}\text { Study of Potential Applications to Ultrafast Electron Diffraction/Microscopy } \\
\text { Using a High Repetition Rate Superconducting Electron Photocathode Gun }\end{array}$ \\
\hline Investigators: & John Byrd \\
\hline
\end{tabular}

Abstract

X-ray free-electron lasers (FELs) currently use radio frequency (RF) photoinjectors. The RF photoinjector has become a reliable source of high-brightness electron beams. Recently, RF guns have supported ultrafast electron diffraction and microscopy (UED/UEM) experiments, thereby demonstrating their ability to produce electron beams with some degree of coherence. A superconducting RF (SRF) gun has been made available to us for testing. We are working with this device in anticipation of an opportunity to build an SRF photoinjector for the Linac Coherent Light Source II high-energy upgrade (LCLS-II HE) to LCLS-II at the Stanford Linear Accelerator Center (SLAC) National Accelerator Laboratory. Furthermore, this photoinjector could also be suitable for UED/UEM applications at Argonne as a complement to existing electron microscopy programs. We have investigated its cryogenic capabilities and compatibility by installing this SRF gun in a concrete shielded bunker that is the primary cavity testing area in the Argonne Physics Division's Accelerator Development and Test Facility (ADTF). In particular, safety aspects of the pressure vessel of the SRF gun cryostat required attention, and adaptations to the ADTF existing cryoplant and transfer line to accommodate the cryostat were considered. 


\section{Argonne}

Laboratory Directed Research and Development

Argonne National Laboratory

9700 South Cass Avenue, Bldg. 201

Argonne, IL 60439

www.anl.gov 